\title{
Asset Prices, Funds' Size and Portfolio Weights in Equilibrium with Heterogeneous and Long-Lived Funds*
}

\author{
Jakša Cvitanić ${ }^{\dagger}$ \\ Semyon Malamud
}

November 19, 2008

\begin{abstract}
We perform a detailed asymptotic analysis of the equilibrium behavior of the asset prices, wealth size and portfolio weights in complete markets equilibria, with long-lived funds. In equilibrium, the fund with the (closest to) log preference will dominate the other funds in size, in the long-run, with probability one. On the other hand, two funds on the opposite sides of the log preference will never dominate each other in expected size. In the very long run, the price behavior of the risky asset will be determined solely by the fund closest to the log preference. However, the price drift and volatility still are affected by higher risk aversions, and the optimal portfolio weights contain a hedging component, positive (negative) for the risk aversions higher (lower) than log. The hedging component is monotone increasing in risk aversion for the times further away from the terminal horizon, but it may become monotone decreasing closer to the terminal horizon. For earlier, but still asymptotically infinite times, the price behavior is impacted also by the funds with risk aversions greater than one. Selling short is never optimal. There are distinct increasing time periods such that the price has the same asymptotic behavior in each period. The long-run per-period return gets lower with time.
\end{abstract}

*The research of J. Cvitanić was supported in part by NSF grants DMS 04-03575 and DMS 06-31298. The research of S. Malamud was supported in part by the National Centre of Competence in Research Financial Valuation and Risk Management (NCCR FINRISK), Project D1 (Mathematical Methods in Financial Risk Management). We are very grateful to Julien Hugonnier, Elyes Jouini, Saša Parad, and to seminar participants at U. of Southern California and U. of Texas at Austin for helpful comments. Existing errors are our sole responsibility.

${ }^{\dagger}$ Caltech, Division of Humanities and Social Sciences, M/C 228-77, 1200 E. California Blvd. Pasadena, CA 91125. Ph: (626) 395-1784. E-mail: cvitanic@hss.caltech.edu

${ }^{\ddagger}$ ETH Zuerich and Swiss Finance Institute. E-mail: semka@math.ethz.ch 
Keywords: Asset pricing; equilibrium; heterogeneous agents

JEL classification: D53, G11, G12 


\section{Introduction}

Consider the simplest possible financial market: a complete market with rational traders consuming only at the end of the time horizon. There are existence results for equilibrium in such a market, ${ }^{1}$ and CAPM type results under well known specific conditions. However, not much is known about specific equilibrium properties. In particular, in this paper we consider the analogue of the benchmark partial equilibrium dynamic model, a la Merton (1971), with the traders having generalized CRRA preferences, and the dividends following a geometric Brownian motion. We find sharp bounds for the asset prices, wealth size and portfolio weights in equilibrium in such financial markets, and study their asymptotic behavior, when the time horizon becomes large. We interpret our traders as long-lived funds which only care about the final consumption.

The main conclusions we reach are the following: the fund with the preference closest to logarithmic will dominate in size other CRRA-like funds in the long-run, with probability one. ${ }^{2}$ However, in terms of expected relative size, two funds, with preferences separated by the log preference, will never dominate each other. In the very long run, the price of the risky asset is impacted solely by the fund closest to the log preferences. Interestingly, its drift and volatility still are impacted by a higher risk aversion. For asymptotically large times, but bounded away from the final horizon, the price behavior is affected by funds more risk averse than log. There are distinct increasing time periods such that the price has the same asymptotic behavior in each period. The long-run per-period return gets lower with time. We also find simple bounds for the portfolio weights of all the funds, which show that selling short never occurs, and that the hedging component is negative (positive) for the risk aversions smaller (larger) than one. Interestingly, the hedging component may become monotone decreasing in risk aversion at the times closer to the end of the investment horizon. For the risk aversions larger than one, we provide explicit limit formulas for the portfolio weights.

Studying equilibrium with heterogeneous funds is hard in general, even in complete markets, as the equilibrium relationship between the market's stochastic discount factor (SDF) and the exogenous total value of the risky assets is very nonlinear. A very nice contribution has been accomplished by Kogan, Ross, Wang and Westerfield (2006) (henceforth KRWW 2006): they consider a market with a CRRA fund and an "irrational" fund that has incorrect beliefs about the drift of the risky asset (there is only one risky asset in the economy). In this framework they show that it is possible to compute the equilibrium analytically. The main message from that paper is that even when the irrational fund does not survive in the

\footnotetext{
${ }^{1}$ See Karatzas and Shreve (1998).

${ }^{2}$ The dominance of the log-fund was also studied in Blume and Easley (1992), and Evstigneev, Hens and Schenk-Hoppe (2006).
} 
long run, it still will have a price impact over a substantial fraction of time, even with very small wealth size, contrary to the common wisdom that there is no long run impact on the price from the funds that vanish. We show that the same effect happens when all the funds are rational - even though only the one closest to the log preference survives in the very long run, the price impact of other funds can be sustained for a long time. This reconfirms the KRWW (2006) message that wealth size is not the same as price impact, the fact illustrated in our case with only rational traders present in the market. ${ }^{3}$

Our results are also related to the so called portfolio turnpikes theorems, which identify conditions, albeit in partial equilibrium, under which the portfolio of an investor converges to a CRRA type portfolio at long horizons. The papers Dybvig, Rogers and Back (1999) and Huang and Zariphopoulou (1999) provide general results of this nature, and further references. A recent paper Yan (2009) studies survival in a model with intermediate consumption, heterogeneous beliefs and heterogeneous discount factors. In particular, he finds that, with heterogeneity only in risk aversion, the agent with the smallest risk aversion will dominate in the long run if the economy is growing. Similarly, Berrada (2008) studies the validity of KRWW (2006) findings in a model with intermediate consumption and finite time horizon. In contrast to KRWW (2006), Berrada (2008) shows that survival and price impact may, in fact, be related - impact on the prices is increasing in the agents' consumption share when the agents have logarithmic preferences. However, when risk aversion is larger than one, that paper is unable to obtain definite conclusions.

While some results in Berrada (2008) are obtained in analytic form, the paper mostly has to rely on numerical simulation. On the other hand, we obtain a very precise analytic picture of what happens in our model: we find, for the case of lognormal cumulative wealth, explicit expressions for the intervals which span the total time horizon, and in each of which the price has the same asymptotic behavior, which we also compute explicitly. In each of the intervals, the asset price process is asymptotically equal to a Black-Scholes, geometric Brownian motion price process. The equilibrium price is asymptotically impacted only by traders with risk aversion no less than one.

In terms of methodological contributions, we show that a lot of sharp results can be obtained without highly sophisticated mathematics - while our proofs are long and technical, they use mostly well known results on inequalities involving expected values. In the process of proving our main theorems, we obtain a lot of auxiliary results, finding bounds on values of expected utility and marginal utility of the traders, on expected values involving wealth size, and on expected values involving the stochastic discount factor. In this latter regard, some of the results are in the spirit of Hansen-Jagannathan (1991) bound on the standard deviation of the SDF. ${ }^{4}$

\footnotetext{
${ }^{3}$ Wang (1996) also studies a model with only rational traders, in a simpler setting.

${ }^{4}$ However, our bounds depend on the market portfolio, and not on individual portfolios.
} 
We also get a simple bound on the asset price volatility: its value has to be larger than the volatility of the total final dividend process, and less than the dividend volatility increased by a factor depending on the difference between the highest and the lowest risk aversion. In other words, in equilibrium, volatility may rise not only due to the volatility of the dividend output, but also due to the difference in risk preferences of the traders. Moreover, we provide exact formulas for the limits of the drift and the volatility of the price, as well as for the optimal portfolio weights. All of these depend on risk aversions larger than one, even at very far away time points. Moreover, with a sufficiently dense set of risk aversions, the optimal portfolio weight, for the risk aversions larger than one, and in the limit, contains a positive hedging component even in the very long run. On the other hand, the hedging component for the risk aversions less than one is no larger than zero. It was also observed in KRWW (2006) that even at time points when the stock price is close to its stationary limit, the portfolio weight is not equal to the corresponding myopic strategy. However, in their framework with two traders, for the time points far enough into the future, the hedging component vanishes. This is not always the case in our model. For example, if the set of the agents' risk aversions is not sufficiently sparse, with high enough heterogeneity there is always a non-zero hedging component. Thus, some of the phenomena we find are completely new and depend on the relative values of the risk aversions of the agents, and hence having many agents in the economy can result in a very different hedging behavior from having two agents only, the case which is usually studied in the literature.

We describe the setup in Section 2, prove the almost sure dominance of the closest to the $\log$ fund in Section 3, provide results on expected relative size in Section 4, compute the long term price behavior in Section 5, and the long term volatility and portfolio behavior in Section 6. Section 7 concludes, while the longer proofs are presented in Appendix.

\section{Setup and notation}

\subsection{The Model}

We consider a standard setting similar to that of Wang (1996). The economy has a finite horizon and evolves in continuous time. Uncertainty is described by a one-dimensional, standard Brownian motion $B_{t}, t \in[0, T]$ on a complete probability space $\left(\Omega, \mathcal{F}_{T}, P\right)$, where $\mathcal{F}$ is the augmented filtration generated by $B_{t}$. There is a single share of a risky asset in the economy, the stock, ${ }^{5}$ which pays a terminal dividend payment

$$
D=D_{T}=e^{\rho T+\sigma B_{T}} .
$$

\footnotetext{
${ }^{5}$ The results of the first part of the paper are easily extended to the case of multiple assets and any complete market model.
} 
We also assume that a zero coupon bond with instantaneous constant risk free rate $r$ is available in zero net supply. ${ }^{6}$ The price of the stock at time $t$ is denoted by $S_{t}$. The instantaneous drift and volatility of the stock price $S_{t}$ are denoted by $\mu_{t}$ and $\sigma_{t}$ respectively,

$$
S_{t}^{-1} d S_{t}=\mu_{t} d t+\sigma_{t} d B_{t}
$$

There are $K$ competitive agents in the economy, which we will also call funds, who behave rationally, and are heterogeneous in risk preferences. Fund $k$ is initially endowed with $\psi_{k}$ shares of the stock at time zero,

$$
\sum_{k} \psi_{k}=1
$$

Fund $k$ 's trading strategy $\pi_{k t}$, the portfolio weight in the risky asset, is assumed to satisfy the standard integrability condition

$$
\int_{0}^{T} \pi_{k t}^{2} \sigma_{t}^{2} d t<\infty
$$

Then, the wealth $W_{k t}$ of fund $k$ evolves as

$$
d W_{k t}=W_{k t}\left(r d t+\pi_{k t}\left(S_{t}^{-1} d S_{t}-r d t\right)\right)
$$

Fund $k$ chooses portfolio strategy $\pi_{k t}$ as to maximize the expected utility

$$
E\left[u_{k}\left(W_{k T}\right)\right]
$$

of its final wealth $W_{k T}$. Utility $u_{k}$ is assumed to be concave, increasing and satisfying the standard Inada conditions

$$
\lim _{x \rightarrow 0} u_{k}^{\prime}(x)=+\infty, \lim _{x \rightarrow \infty} u_{k}^{\prime}(x)=0
$$

\subsection{The Equilibrium}

Definition 2.1 We say that the market is in equilibrium if the funds behave optimally and both the risky asset market and the risk-free market clear.

It is well known that the above financial market is complete, if the volatility process $\sigma_{t}$ of the stock price is almost everywhere strictly positive. ${ }^{7}$ When the market is complete, there exists a unique stochastic discount factor (SDF) $M=M_{T}$ such that the stock price is given by

$$
S_{t}=e^{r(t-T)} \frac{E_{t}[M D]}{E_{t}[M]} .
$$

\footnotetext{
${ }^{6}$ The assumption of constant $r$ is introduced only for simplicity of exposition.

${ }^{7}$ This follows for example from the results of Anderson and Raimondo (2008). In the CRRA setting, it will follow from Proposition 6.1 below.
} 
Since $u_{k}$ satisfies Inada conditions, the inverse of the marginal utility

$$
I_{k}(x):=\left(u_{k}^{\prime}\right)^{-1}(x)
$$

is strictly positive and it is well known (see, for example, Cvitanić and Zapatero (2004)) that the optimal terminal wealth is then of the form

$$
W_{k T}=I_{k}\left(\lambda_{k} M\right)
$$

where $\lambda_{k}$ is determined via the budget constraint

$$
E\left[I_{k}\left(\lambda_{k} M\right) M\right]=W_{k 0}=\psi_{k} S_{0}=\psi_{k} E[D M] .
$$

Because of the market completeness, equilibrium allocation is Pareto-efficient and can be characterized as an Arrow-Debreu equilibrium. See, e.g. Duffie (1986), Wang (1996). Because the endowments are co-linear (all agents hold shares of the same single stock), the equilibrium is in fact unique, up to a multiplicative factor, and unique if we fix the risk-free rate. See, e.g., Dana (1995), Dana (2001). ${ }^{8}$ We formalize this in

Proposition 2.1 The equilibrium allocation is given by

$$
W_{k T}=I_{k}\left(\lambda_{k} M\right), E\left[I_{k}\left(\lambda_{k} M\right) M\right]=\psi_{k} E[D M]
$$

and equilibrium SDF $M$ solves the equation

$$
\sum_{k} I_{k}\left(\lambda_{k} M\right)=D
$$

The following lemma is a direct consequence of (2).

Lemma 2.1 For any fund $k$, the equilibrium SDF satisfies

$$
M \geq \max _{k}\left\{\lambda_{k}^{-1} u_{k}^{\prime}(D)\right\}
$$

Proof. We have

$$
D=\sum_{k} I_{k}\left(\lambda_{k} M\right)>I_{k}\left(\lambda_{k} M\right)
$$

and the claim follows by applying $u_{k}^{\prime}$ to both sides of the inequality.

This lemma will be crucial for most bounds derived in this paper. Note that $\lambda_{k}^{-1} u_{k}^{\prime}(D)$ is, up to a constant, the SDF in the economy populated by fund $k$ only. In fact, for a particular choice of the risk-free rate, it will be equal to the SDF. In this regard, Lemma 2.1 may be interpreted as saying that the price of a state in the heterogeneous economy dominates the price in particular homogeneous economies.

\footnotetext{
${ }^{8}$ Since the endowment is neither bounded away from zero nor from infinity, some additional care is needed to show the existence of equilibrium. See, e.g., Dana (2001) and Malamud (2008a).
} 


\section{Almost sure extinction relative to the log fund}

\subsection{Almost sure convergence of relative fund size}

It is well known that, in typical financial market models, the fund that maximizes the logutility from final wealth also maximizes the long-run growth rate. It is then to be expected that the log-fund will dominate the market in the long-run. This is the case in KRWW (2006), where the other fund is not rational, but it is also the case in our market, with rational funds and a standard choice for $D$, as we will now show.

Introduce

$$
\gamma_{k}(x):=-\frac{x u_{k}^{\prime \prime}(x)}{u_{k}^{\prime}(x)}
$$

the (relative) risk aversion of fund $k$, and denote by

$$
b_{k}(x):=\frac{1}{\gamma_{k}(x)}
$$

its relative risk tolerance. Intuitively, the only thing that matters for the long run behavior of the optimal wealth is the risk attitude of the fund for very small and very large wealth. If the risk aversion starts oscillating as the wealth approaches zero/infinity, the optimal behavior of a fund may become quite strange. To avoid this, we impose the following natural

Assumption 3.1 The limits

$$
\gamma_{i}^{0}:=\lim _{x \downarrow 0} \gamma_{i}(x), \gamma_{i}^{\infty}:=\lim _{x \rightarrow \infty} \gamma_{i}(x)
$$

exist and are strictly positive.

That is, for very small and very large wealth, the fund behaves as a CRRA investor, but otherwise we allow for a wealth-dependent risk aversion. The class of utilities, satisfying Assumption 3.1 is quite large.

For technical reasons, we also impose the following assumption for each fund, which supposes consistency in risk aversion across wealth size relative to the log preference:

Assumption 3.2 For every fund $i$ in the economy, either $\gamma_{i}^{0}, \gamma_{i}^{\infty}>1$ or $\gamma_{i}^{0}, \gamma_{i}^{\infty}<1$ or $\gamma_{i}^{0}=\gamma_{i}^{\infty}=1$.

We expect the fund which is the closest to the log preference to have the highest long term growth rate and dominate all others in the long run. The following theorem makes this intuition rigorous. 
Theorem 3.1 Assume Assumptions 3.1 and 3.2 and

$$
D=e^{\rho T+\sigma B_{T}} .
$$

Suppose that there exists a unique fund $i=0$ closest to being a log fund, that is, we assume:

$$
\max \left\{\left|\gamma_{0}^{0}-1\right|,\left|\gamma_{0}^{\infty}-1\right|\right\}<\min _{i \neq 0} \min \left\{\left|\gamma_{i}^{0}-1\right|,\left|\gamma_{i}^{\infty}-1\right|\right\}
$$

Then, in equilibrium,

$$
\lim _{T \rightarrow \infty} \frac{W_{i T}}{W_{0 T}}=0
$$

almost surely for any $i \neq 0$.

Assumption 3.1 is related to the portfolio turnpike theorems; see, e.g., Dybvig, Rogers and Back (1999) and Huang and Zariphopoulou (1999). They show that, if the risk aversion is asymptotically constant at large wealth levels and the stock prices are growing, then the optimal portfolio will converge at a long horizon to that of the investor with a CRRA utility. In a sense, Theorem 3.1, and further results below, can be viewed as equilibrium analogs of the turnpike theorems.

The proof of this intuitive result is rather non-trivial and is based on several useful bounds for the funds wealth. The most important one (see Lemma A.3 in Appendix) shows that, in equilibrium, for fund $i$ and for some constants $K_{1}, K_{2}$ independent of $T$ and independent of the aggregate endowment $D$, we have

$$
K_{1} \leq \frac{E\left[W_{i T} u_{i}^{\prime}\left(W_{i T}\right)\right]}{E\left[D u_{i}^{\prime}(D)\right]} \leq K_{2}
$$

Because the market is complete, the marginal utility $u_{i}^{\prime}\left(W_{i T}\right)$ of fund $i$ 's wealth is in fact proportional to the unique SDF. The quantity $E\left[W_{i T} u_{i}^{\prime}\left(W_{i T}\right)\right]$ is thus (proportional to) the value of fund $i$ 's wealth. On the other hand, $E\left[D u_{i}^{\prime}(D)\right]$ is (proportional to) the value of the aggregate endowment in an artificial economy, populated only by fund $i$. The above bounds show that, in equilibrium, the two values have a comparable behavior. When the fund has a CRRA utility, the inequality shows that its expected utility is bounded from below and from above by a multiple of the expected utility of a fund that consumes the total dividend output $D$ in the economy.

We have obtained also results on the almost sure convergence of the relative size of any two funds in Proposition 4.2 below. We present that result later because its proof requires some additional estimates from the next section.

\section{Expected relative size of funds}

\subsection{Bounds on expected relative size of funds}

The main result we want to show in this subsection is 
Proposition 4.1 Let $i, j$ be funds with risk aversions $\gamma_{i}(x), \gamma_{j}(x)$. Then,

(i) If either $\gamma_{i}(x) \geq \gamma_{j}(y) \geq 1$ for all $x$, $y$ or $\gamma_{i}(x) \leq \gamma_{j}(y) \leq 1$, that is, fund $j$ is closer to being log fund than fund $i$, then

$$
E\left[\frac{W_{i T}}{W_{j T}}\right] \leq \frac{W_{i 0}}{W_{j 0}}
$$

(ii) If $\gamma_{j}(x) \equiv 1$ then

$$
E\left[\frac{W_{i T}}{W_{j T}}\right]=\frac{W_{i 0}}{W_{j 0}}
$$

(iii) If either $\gamma_{i}(x) \leq 1 \leq \gamma_{j}(x)$ or $\gamma_{j}(x) \leq 1 \leq \gamma_{i}(x)$ for all $x$ then

$$
E\left[\frac{W_{i T}}{W_{j T}}\right] \geq \frac{W_{i 0}}{W_{j 0}}
$$

Result (ii) seems surprising at first, given that in the previous section we showed that the (closest to) log fund will dominate in the long run with probability one. Here, however, the expected relative wealth of other funds relative to the log fund remains constant: log fund will always own "on average" the same fraction of the economy at time $T$ as at time zero. This is actually well known, and is no longer surprising if we recall that the log fund's wealth process is proportional to the inverse $1 / M_{t}$ of the SDF process, and that $W_{j t} M_{t}$ is a martingale for any wealth process $W_{j t}$. For example, if the ratio of the wealth of the two funds is proportional to the martingale $\exp \left\{-t \sigma^{2} / 2+\sigma B_{t}\right\}$, then the expected value is constant, but this random variable converges to zero as $t \rightarrow \infty$, with probability one.

From (iii) we see that if the risk aversion of fund $i$ is on the other side of value 1 (the log preference) than the fund $j$ 's risk aversion, then the ratio of the two funds' wealth will not decrease in expected value as $T \rightarrow \infty$, no matter how close one of the funds is to being a $\log$ fund. Expected value of the relative wealth sizes of two funds separated by the log fund is bounded away from zero.

The key to the result of Proposition 4.1 is the following

Lemma 4.1 We have

$$
E\left[\frac{W_{i T} W_{i 0}^{-1}}{W_{j T} W_{j 0}^{-1}}\right]-1=-\frac{1}{W_{i 0}} \operatorname{Cov}\left(\frac{W_{i T}}{W_{j T}}, M W_{j T}\right) .
$$

Proof of Lemma 4.1. The claim follows by direct calculation from the identities

$$
W_{i 0}=E\left[M W_{i T}\right], W_{j 0}=E\left[M W_{j T}\right]
$$


This result has a clear risk premium structure. The left-hand side is the expected return of fund $i$ over $[0, T]$ relative to the return of fund $j$ and Lemma 4.1 shows that the excess relative return is given by the covariance of the relative return with the SDF-weighted wealth of fund $j$. For example, if $i$ is less risk averse, the dependence of $W_{i T}$ on $M$ is steeper and hence the ratio $W_{i T} / W_{j T}$ is decreasing in $M$. Depending on whether $j$ is more or less risk averse than $\log , M W_{i T}$ will be either increasing or decreasing in $M$, determining the sign of the covariance.

A detailed proof of Proposition 4.1 is provided in Appendix and is also based on the following known

Lemma 4.2 If both $g(x)$ and $h(x)$ are increasing (or both decreasing) then

$$
E[g(Z)] E[h(Z)] \leq E[g(Z) h(Z)]
$$

If both $g, h$ are strictly increasing (or both strictly decreasing), then the inequality is also strict unless $Z$ is constant almost surely. If one function is increasing and the other is decreasing, then the inequality reverses.

\subsection{Convergence of expected relative size}

From now on we assume that all funds have constant relative risk aversion (CRRA) utilities. ${ }^{9}$ The main result of this subsection is the following

Theorem 4.1 If either $\gamma_{i}<\gamma_{j}<1$ or $\gamma_{i}>\gamma_{j}>1$ then

$$
E\left[W_{i T} / W_{j T}\right] \rightarrow 0
$$

Thus, as long as two CRRA funds are on the same side of the log preference, the one closer to the log will dominate in expectation in the long run. Since the wealth is nonnegative, standard Chebyshev inequality implies that it also converges to zero in probability.

When risk aversion is homogeneous across agents, the equilibrium SDF is explicitly determined by $D^{-\gamma} / E\left[D^{-\gamma}\right]$. But, when risk aversion is heterogeneous, SDF is a solution of a highly non-linear equation and no explicit solution is possible, except for some very special values of risk aversion; see, for example, Wang (1996). In the lemma below, we establish strong bounds on the equilibrium SDF.

\footnotetext{
${ }^{9}$ Most of the analysis can be extended to more general utility functions as in the previous sections, but it becomes overly technical.
} 
Lemma 4.3 Let $\Gamma \geq 1$ be such that $\Gamma b_{i}>1$ for all $i$ and $\gamma \leq 1$ be such that $\gamma b_{i} \leq 1$ for all i. Then,

$$
\begin{aligned}
\left(\sum_{i} D^{-\gamma_{i} / \gamma}\left(\psi_{i} E[D M] / E\left[M^{1-b_{i}}\right]\right)^{\gamma_{i} / \gamma}\right)^{\gamma} & \\
\leq M & \leq\left(\sum_{i} D^{-\gamma_{i} / \Gamma}\left(\psi_{i} E[D M] / E\left[M^{1-b_{i}}\right]\right)^{\gamma_{i} / \Gamma}\right)^{\Gamma} .
\end{aligned}
$$

The quantity $D^{-\gamma_{i}}\left(\psi_{i} E[D M] / E\left[M^{1-b_{i}}\right]\right)^{\gamma_{i}}$ can be viewed as the "individual" SDF in an economy populated only by fund $i$. It is known that, when risk aversion is heterogeneous, the equilibrium SDF can be represented as a generalized weighted Hölder average of the "individual" SDFs (see, e.g., Malamud (2008a), Malamud (2008b), Jouini and Napp (2008), Shefrin (2005)). Lemma 4.3 shows that $M$ can be estimated from both below and above by Hölder averages with different exponents $\gamma$ and $\Gamma$.

The weights $\left(\psi_{i} E[D M] / E\left[M^{1-b_{i}}\right]\right)^{\gamma_{i}}$ are not directly helpful for getting good bounds for $M$. The following useful lemma allows us to obtain uniform bounds for these weights. It has a very clear economic meaning: the maximal utility of a fund is larger than the utility from simply consuming its endowment, and is smaller than the utility from consuming the aggregate endowment of the economy.

Lemma 4.4 Let $M$ be the equilibrium SDF. If $\gamma_{i}<1$ then

$$
1 \leq \frac{E[D M]^{1-\gamma_{i}} E\left[M^{1-b_{i}}\right]^{\gamma_{i}}}{E\left[D^{1-\gamma_{i}}\right]} \leq \psi_{i}^{\gamma_{i}-1} .
$$

If $\gamma_{i}>1$ then

$$
\psi_{i}^{\gamma_{i}-1} \leq \frac{E[D M]^{1-\gamma_{i}} E\left[M^{1-b_{i}}\right]^{\gamma_{i}}}{E\left[D^{1-\gamma_{i}}\right]} \leq 1 .
$$

Proof: The utility of fund $i$ 's optimal wealth is given by

$$
\begin{array}{rl}
\frac{1}{1-\gamma_{i}} E\left[W_{i T}^{1-\gamma_{i}}\right]=\frac{1}{1-\gamma_{i}} \psi_{i}^{1-\gamma_{i}}\left(\frac{E[D M]}{E\left[M^{1-b_{i}}\right]}\right)^{1-\gamma_{i}} & E\left[M^{1-b_{i}}\right] \\
& =\frac{1}{1-\gamma_{i}} \psi_{i}^{1-\gamma_{i}} E[D M]^{1-\gamma_{i}} E\left[M^{1-b_{i}}\right]^{\gamma_{i}} .
\end{array}
$$

The utility from just consuming his endowment (the terminal dividend of his initial portfolio) is

$$
\frac{1}{1-\gamma_{i}} E\left[\left(\psi_{i} D\right)^{1-\gamma_{i}}\right]=\frac{1}{1-\gamma_{i}} \psi_{i}^{1-\gamma_{i}} E\left[D^{1-\gamma_{i}}\right] .
$$

Furthermore, by definition, in equilibrium we must have $W_{i T} \leq D$ and therefore

$$
\frac{1}{1-\gamma_{i}} E\left[\left(\psi_{i} D\right)^{1-\gamma_{i}}\right] \leq \frac{1}{1-\gamma_{i}} E\left[W_{i T}^{1-\gamma_{i}}\right] \leq \frac{1}{1-\gamma_{i}} E\left[D^{1-\gamma_{i}}\right]
$$


Multiplying both sides by $1-\gamma_{i}$ and using (5), we get the result.

Lemmas 4.3 and 4.4 together allow us to obtain good bounds on the ratio $W_{i T} / W_{j T}$ and prove Theorem 4.1. The details are contained in Appendix.

\subsubsection{Almost sure convergence of relative size}

We go back now to the almost sure convergence of relative size of two given funds. By Theorem 3.1, the fund closest to log dominates all others almost surely. One could then expect that Theorem 3.1 can be extended along the lines of Theorem 4.1: if $\gamma_{m}$ is closer to one than $\gamma_{m}$, then $W_{m T} / W_{k T}$ might be expected to converge to zero almost surely. However, in general, the opposite may be true in case $\gamma_{k}<1<\gamma_{m}$, as the second part of the following proposition shows.

Proposition 4.2 Suppose that one of the following conditions holds true:

- $\gamma_{i}<\gamma_{j}<1$

- $\gamma_{i}>\gamma_{j}>1$

- $\gamma_{i}<1<\gamma_{j}$ and

$$
\left(1-\gamma_{i}\right)^{2} b_{i}-\left(1-\gamma_{j}\right)^{2} b_{j}-\left(1-\gamma_{k}\right)^{2}\left(b_{i}-b_{j}\right)>0
$$

for at least one $k$. (In particular, this is the case if $1-\gamma_{i}>\gamma_{j}-1$, by setting $k=i$ of $k=j$.) Then, $W_{i T} / W_{j T} \rightarrow 0$ almost surely. On the other hand, if $\gamma_{i}<1<\gamma_{j}$ and

$$
\left(1-\gamma_{i}\right)^{2} b_{i}-\left(1-\gamma_{j}\right)^{2} b_{j}-\left(1-\gamma_{k}\right)^{2}\left(b_{i}-b_{j}\right)<0
$$

for all $k$, then, $W_{i T} / W_{j T} \rightarrow \infty$ almost surely (even if $\gamma_{i}$ may be closer to one than $\gamma_{j}$ ).

KRWW (2006) show that, in the case of two funds, the fund with preferences closest to log will dominate the other fund almost surely. Proposition 4.2 shows that this is not any more true when there are more than two agents: it might happen that an agent with preferences closer to log (but less than one) will experience extinction relative to a fund which is further away from log.

\section{Long-term price behavior}

In the previous two sections, we have obtained a detailed picture of the asymptotic behavior of the wealth of different funds. In particular, we have seen that the fund closest to the log dominates in the long run and owns the whole economy. Intuitively, one expects that the long run behavior of the stock price is also determined solely by the fund closest to the log. As we will see in this section, this is only true to some extent. We will need the following 
Assumption 5.1 The funds have CRRA preferences and there exists a unique fund, denoted by 0 , the risk aversion of which is closest to one.

Let $\gamma_{0}$ be the risk aversion closest to one. Let us reorder the risk aversions of funds in the economy as, for some fixed $k, l$,

$$
\gamma_{-k}<\gamma_{-k+1}<\cdots<\gamma_{-1}<\gamma_{0}<\gamma_{1}<\cdots<\gamma_{l}
$$

Note that we have $\gamma_{-1}<1$, otherwise $1 \leq \gamma_{-1}<\gamma_{0}$ would imply that $\gamma_{-1}$ is closer to 1 . Similarly, $\gamma_{1}>1$, otherwise $\gamma_{0}<\gamma_{1}<1$ would imply that $\gamma_{1}$ is closer to one. If $\gamma_{0}<1$ then, by definition

$$
\left|1-\gamma_{0}\right|<\left|1-\gamma_{1}\right| \Leftrightarrow 1-\gamma_{0}<\gamma_{1}-1 \Leftrightarrow \gamma_{0}+\gamma_{1}>2
$$

Throughout this section we will let

$$
t=\lambda T
$$

for some fixed $\lambda$, so that when we let $T \rightarrow \infty$, then also $t \rightarrow \infty$.

We use the notation $X_{t} \sim Y_{t}$ to mean that $X_{t} / Y_{t} \rightarrow 1$ as $t \rightarrow \infty$. The main result of this section is

Theorem 5.1 Assume $D=e^{\rho T+\sigma B_{T}}$. Define the intervals

$$
\begin{gathered}
\Pi_{l}=\left(0, \frac{2}{\gamma_{l}+\gamma_{l-1}}\right), \\
\Pi_{0}=\left(\frac{2}{\gamma_{0}+\gamma_{1}}, 1\right)
\end{gathered}
$$

and

$$
\Pi_{i}=\left(\frac{2}{\gamma_{i}+\gamma_{i+1}}, \frac{2}{\gamma_{i-1}+\gamma_{i}}\right)
$$

for $i=1, \cdots, l-1$. Fix $i \in\{1, \cdots, l\}$ and $\lambda \in \Pi_{i}$. Then, there exists a deterministic function $f_{i}(T)$ satisfying

$$
\psi_{i}^{-1} \min \left\{\psi_{0}, \psi_{0}^{\gamma_{0}}\right\} \leq f_{i}(T) \leq \psi_{i}^{-\gamma_{i}} \max \left\{\psi_{0}, \psi_{0}^{\gamma_{0}}\right\}
$$

for all $T$ and such that

$$
S_{t} \sim P_{i t}:=e^{r\left(1-\lambda^{-1}\right) t} f_{i}\left(t \lambda^{-1}\right) e^{\rho t \lambda^{-1}} e^{\sigma\left(1-\gamma_{0}+\gamma_{i}\right) B_{t}} e^{\frac{1}{2} \sigma^{2} t \lambda^{-1}\left(\left(\left(1-\gamma_{0}\right)^{2}-\gamma_{i}^{2}\right)(1-\lambda)+\left(1-\gamma_{i}\right)^{2}-\left(1-\gamma_{0}\right)^{2}\right)} .
$$

If $\lambda \in \Pi_{0}$, that is

$$
\lambda>\frac{2}{\gamma_{0}+\gamma_{1}}
$$

then

$$
S_{t} \sim P_{0 t}:=D_{t} e^{r\left(1-\lambda^{-1}\right) t} e^{\rho t\left(\lambda^{-1}-1\right)} e^{\frac{1}{2} \sigma^{2} t \lambda^{-1}\left(1-2 \gamma_{0}\right)(1-\lambda)}=e^{r(t-T)} \frac{E_{t}\left[D^{1-\gamma_{0}}\right]}{E_{t}\left[D^{-\gamma_{0}}\right]}
$$


Furthermore, the price process satisfies $\left(P_{i, \lambda_{i} T} e^{-r\left(\lambda_{i}^{-1}-1\right) T}\right) /\left(P_{i+1, \lambda_{i+1} T} e^{-r\left(\lambda_{i+1}^{-1}-1\right) T}\right) \rightarrow+\infty$ as $T \rightarrow \infty$ for any $\lambda_{i}, \lambda_{i+1} \in \Pi_{i}, \Pi_{i+1}$, respectively. In particular, $\left(P_{i, \lambda_{i} T}\right) /\left(P_{i+1, \lambda_{i+1} T}\right) \rightarrow+\infty$.

Equation (9) shows that in the very long run, that is, for $t$ sufficiently close to $T$, and $T \rightarrow \infty$, there will be no longer any price impact from other funds, and the price will be completely determined as if there is only the (closest to) log fund in the market. By Theorem 3.1, all funds except for (closest to) log fund vanish in the long run and therefore the price impact of these funds should also vanish. Theorem 5.1 shows that this vanishing takes place gradually. Agent $i$ with risk aversion above one impacts the price precisely in the interval $\Pi_{i}$ and his price impact vanishes precisely when $t / T$ crosses from $\Pi_{i}$ to $\Pi_{i-1}$. Moreover, the price behavior changes dramatically when we go from $\Pi_{i}$ to $\Pi_{i-1}$. A similar phenomenon has been discovered by KRWW (2006) in the context of heterogeneous beliefs. They show that, even if the irrational trader disappears in the long run, he may generate price impact for a substantial fraction of time. The gradual structure of multiple intervals that we obtain here, with the agents' impact vanishing one after another, seems to be new in the literature.

Surprisingly, the agents with risk aversion below one have no price impact at all, asymptotically. One reason may be due to the fact that these agents "over-leverage", as discussed next: For long horizons, the (closest to) log fund simply invests all its wealth into the risky asset (see Theorem 6.2 below). Not surprisingly (as shown by Proposition 6.2 below), the agents with risk aversion smaller than one invest more into the risky asset than the log fund, that is, they borrow via the risk-free market from the agents with risk aversion above one. In order that the leveraged agents can repay the debt to the agents with risk aversion above one, as indicated by Proposition 4.2 their terminal consumption may become small at a faster rate than for the other agents, and they do not generate any price impact.

We do not know the exact asymptotic behavior of the functions $f_{i}(T)$. However, since we know that they are bounded, we can explicitly calculate the long run per-period stock return.

Corollary 5.1 Let $\lambda \in \Pi_{i}$. The long run per-period expected return is given by

$$
\begin{aligned}
R(\lambda) & :=\lim _{T \rightarrow \infty}(T-t)^{-1} \log \left(\frac{E_{t}[D]}{S_{t}}\right) \\
& =\frac{1}{2} \sigma^{2}+r-\frac{1}{2} \sigma^{2}(1-\lambda)^{-1}\left(\left(\left(1-\gamma_{0}\right)^{2}-\gamma_{i}^{2}\right)(1-\lambda)+\left(1-\gamma_{i}\right)^{2}-\left(1-\gamma_{0}\right)^{2}\right) .
\end{aligned}
$$

In particular, $R(\lambda)$ is monotone decreasing and continuous in $\lambda$.

Figure 1 shows the graph of the expected return for the remaining period at times $t=\lambda T$. Even though the graph looks quite smooth, there are actually kinks at the boundaries between intervals $\Pi_{i}$, which would be visible if that portion of the graph was enlarged. 


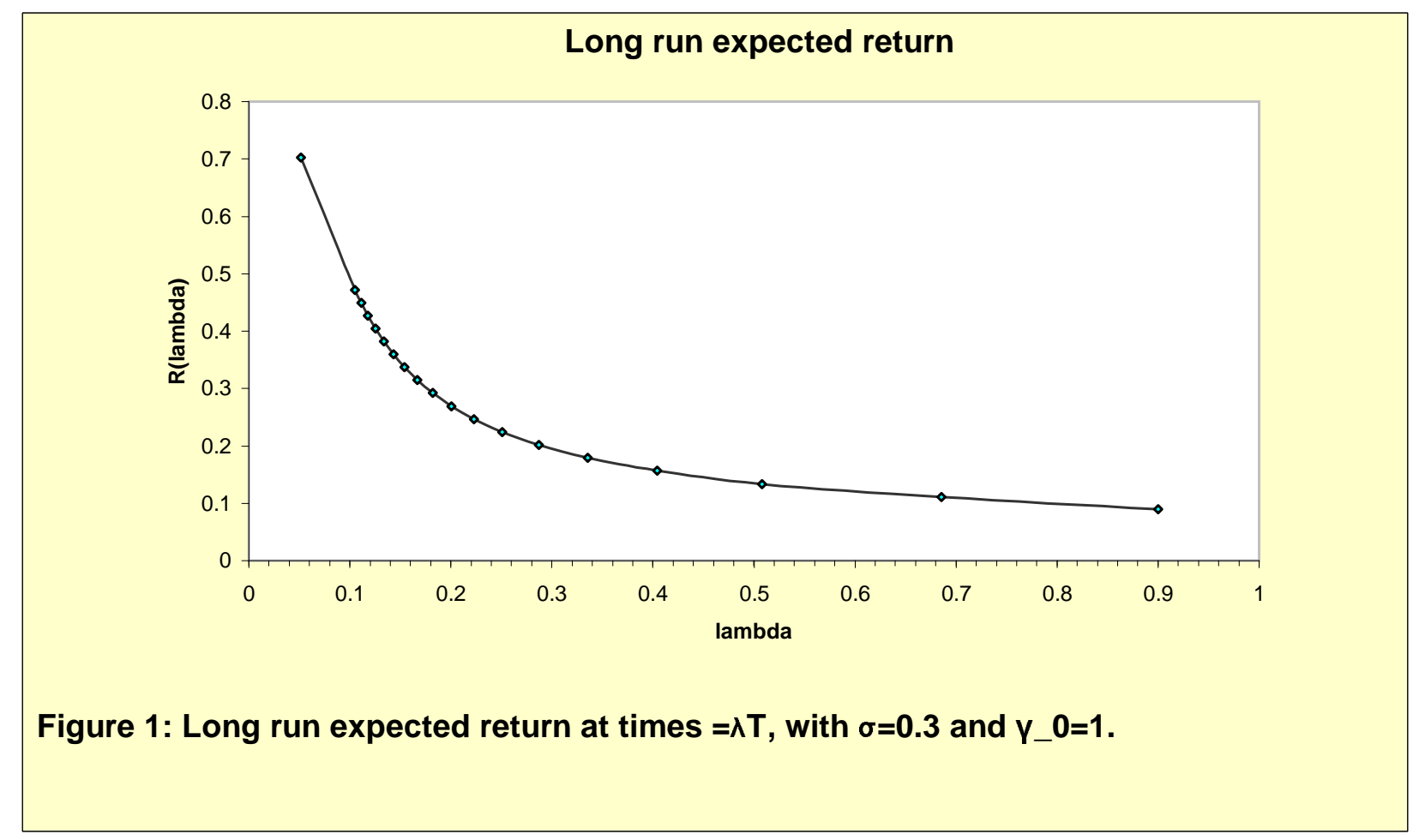

We complete the section with some interesting global bounds for the stock price. Recall that $\gamma_{-k}$ and $\gamma_{l}$ are the minimal and maximal risk aversion respectively. Note that in the homogeneous economy populated by only one fund with risk aversion $\gamma$ the stock price is given by $S_{t}=e^{r(t-T)} \frac{E_{t}\left[D^{1-\gamma}\right]}{E_{t}\left[D^{-\gamma}\right]}$. We now show that in the multi-fund economy the price is bounded between such quantities corresponding to the lowest and the highest risk aversion:

Proposition 5.1 We have

$$
e^{r(t-T)} \frac{E_{t}\left[D^{1-\gamma_{l}}\right]}{E_{t}\left[D^{-\gamma_{l}}\right]} \leq S_{t} \leq e^{r(t-T)} \frac{E_{t}\left[D^{1-\gamma_{-k}}\right]}{E_{t}\left[D^{-\gamma_{-k}}\right]}
$$

This result has an interesting connection with the notion of bubbles and panics, introduced by Cao and Ou-Yang (2005). By their definition, a bubble (panic) occurs if the price is above (below) the maximal (minimal) "individual" prices $S_{t}=e^{r(t-T)} \frac{E_{t}\left[D^{\left.1-\gamma_{i}\right]}\right.}{E_{t}\left[D^{-\gamma_{i}}\right]}$. Proposition 5.1 shows that neither bubbles nor panics occur in our setting. However, interestingly, for models with consumption, the situation is different, and both bubbles and panics may occur; see Malamud (2008b).

\section{Portfolio Strategies}

In this section we analyze the equilibrium drift and volatility of the risky asset, as well as the optimal portfolios, and study their asymptotic behavior. 


\subsection{Equilibrium bounds on portfolio weights}

Denote by $\pi_{\log t}$ the portfolio weight of the $\log$ fund (which is not necessarily present in the economy) and, as before, by $\pi_{i t}$ the portfolio weight of fund $i$. The first main result in this subsection is:

Proposition 6.1 The price volatility is always larger than the dividend volatility, and bounded from above as follows, for all t:

$$
\sigma \leq \sigma_{t} \leq \sigma\left(1+\max _{i} \gamma_{i}-\min _{i} \gamma_{i}\right)
$$

The instantaneous Sharpe ratio satisfies

$$
\sigma \min _{i} \gamma_{i} \leq \frac{\mu_{t}-r}{\sigma_{t}} \leq \sigma \max _{i} \gamma_{i}
$$

and therefore

$$
\frac{\min _{i} \gamma_{i}}{1+\max _{i} \gamma_{i}-\min _{i} \gamma_{i}} \leq \pi_{\text {logt }}=\frac{\mu_{t}-r}{\sigma_{t}^{2}} \leq \max _{i} \gamma_{i}
$$

The bounds (12) are instantaneous analogs of the bounds of Proposition 5.1. Namely, in the "individual" economy of agent $i$, the Sharpe ratio is given by $\sigma \gamma_{i}$, while, when the risk aversions are heterogeneous, it stays between the minimal and the maximal risk aversion multiplied by $\sigma$. As we will see below (Theorem 6.1), both bounds (11) and (12) are asymptotically sharp when risk aversion is larger than one.

The bounds (11) have direct implications for the well known volatility puzzle, that is, that, empirically, the volatility of the stock prices is significantly higher than the volatility of the dividends. When inequalities (11) become sharp, they show that heterogeneous risk aversion drives the price volatility up. The size of the ratio $\sigma_{t} / \sigma$ is then determined by the size $\max _{i} \gamma_{i}-\min _{i} \gamma_{i}$ of heterogeneity.

Recall that, when both drift and volatility of $S_{t}$ are constant, the optimal partial equilibrium portfolio is myopic (i.e., it only depends on instantaneous stock returns), and is given by

$$
\pi_{i t}^{\text {myopic }}=\frac{\mu_{t}-r}{\gamma_{i} \sigma_{t}^{2}}=\gamma_{i}^{-1} \pi_{\text {logt }} .
$$

When risk aversion is heterogeneous, both drift and volatility of $S_{t}$ are stochastic and it is generally not possible to find closed form solutions for $\pi_{i t}$. The next proposition provides bounds for equilibrium optimal portfolios.

Proposition 6.2 We have:

(1) if $\gamma_{i}>\gamma_{j}>1$ then $\pi_{i t}<\pi_{j t}$; 
(2) in general,

$$
\begin{aligned}
& \pi_{\text {logt }}<\pi_{i t}<\pi_{i t}^{\text {myopic }} \text { if } \gamma_{i}<1 \\
& \pi_{\text {logt }}>\pi_{i t}>\pi_{i t}^{\text {myopic }} \text { if } \gamma_{i}>1 .
\end{aligned}
$$

The fact that the stock holding is monotone decreasing in risk aversion is intuitively clear. Interestingly enough, the proof of this result is non-trivial and we do not know whether this monotonicity also holds for risk aversions below one.

We can always decompose

$$
\pi_{i t}=\pi_{i t}^{\text {myopic }}+\pi_{i t}^{\text {hedging }}
$$

where $\pi_{i t}^{\text {hedging }}$ is the hedging component, arising because the investment opportunity set is stochastic. Proposition 6.2 shows that the hedging component $\pi^{\text {hedging }}$ is always positive (negative) for risk aversion above (below) one. Furthermore, by Proposition 6.1, $\pi_{\text {logt }}>0$ and therefore (13)-(14) imply that there is no short-selling by any fund and moreover

$$
\pi_{i t} \leq \max \left\{\max _{i} \gamma_{i}, \max _{i} \gamma_{i} / \min _{i} \gamma_{i}\right\}
$$

To prove the above results, we will use the notion of a representative agent. Since the market is complete, it is well known (see, e.g., Cvitanić and Zapatero 2004) that the prices in our heterogeneous economy coincide with those in an artificial economy, populated by a single, representative fund with a utility function $U$, and the equilibrium stochastic discount factor equals the marginal utility of the representative fund, evaluated at the aggregate endowment,

$$
M=U^{\prime}(D) \text {. }
$$

That is, the function $U^{\prime}(x)$ is the unique solution to the equation

$$
\sum_{i} \psi_{i} E[D M] E\left[M^{1-b_{i}}\right]^{-1}\left(U^{\prime}(x)\right)^{-b_{i}}=x .
$$

Let

$$
\gamma^{U}(x)=-\frac{x U^{\prime \prime}(x)}{U^{\prime}(x)}
$$

be the risk aversion of the representative fund.

Proposition 6.3 The relative risk aversion $\gamma^{U}(x)$ is monotone decreasing in $x$ and satisfies

$$
\max _{i} \gamma_{i}=\lim _{x \rightarrow+0} \gamma^{U}(x) \geq \gamma^{U}(x) \geq \lim _{x \rightarrow \infty} \gamma^{U}(x)=\min _{i} \gamma_{i}
$$

In the case of a one period economy, Proposition 6.3 was proved by Benninga and Mayshar (2000). The proof for our continuous time economy is analogous to theirs, and we present it in Appendix for the reader's convenience.

It turns out that the stock volatility and the optimal portfolio weights can be expressed in terms of conditional expected values involving $M, D$ and $\gamma^{U}(D)$, as follows: 
Proposition 6.4 The drift and volatility of the stock price are given by

$$
\mu_{t}=r+\sigma \frac{E_{t}\left[M \gamma^{U}(D)\right]}{E_{t}[M]} \sigma_{t}, \sigma_{t}=\sigma\left(1-\frac{E_{t}\left[M D \gamma^{U}(D)\right]}{E_{t}[M D]}+\frac{E_{t}\left[M \gamma^{U}(D)\right]}{E_{t}[M]}\right)
$$

and the optimal portfolio of agent $i$ is given by

$$
\pi_{i t}=\frac{\left(b_{i}-1\right) \frac{E_{t}\left[M^{1-b_{i}} \gamma^{U}(D)\right]}{E_{t}\left[M^{1-b_{i}}\right]}+\frac{E_{t}\left[M \gamma^{U}(D)\right]}{E_{t}[M]}}{1-\frac{E_{t}\left[M D \gamma^{U}(D)\right]}{E_{t}[M D]}+\frac{E_{t}\left[M \gamma^{U}(D)\right]}{E_{t}[M]}}
$$

Proposition 6.1 follows directly from Proposition 6.4 and (17). Furthermore, by Proposition 6.3, the representative fund has the decreasing relative risk aversion (DRRA) property. This fact plays a crucial role in the proof of the following

Lemma 6.1 We have

$$
\frac{E_{t}\left[M \gamma^{U}(D)\right]}{E_{t}[M]} \geq \frac{E_{t}\left[M D \gamma^{U}(D)\right]}{E_{t}[M D]}
$$

Furthermore,

$$
\frac{E_{t}\left[M^{1-x} \gamma^{U}(D)\right]}{E_{t}\left[M^{1-x}\right]}
$$

is monotone decreasing in $x$.

Now, Proposition 6.2 follows by combining Lemma 6.1 and Proposition 6.4. See Appendix for the remaining proofs.

\subsection{Long run drift and volatility of the stock price}

In Theorem 5.1, equation (8), we have found the approximate behavior of the stock price $S_{t}$ at $t=\lambda T$ as $T \rightarrow \infty$. In this section we find the exact limit of the drift and the volatility of the price and show that it may differ substantially from what could be expected from Theorem 5.1.

Denote by $J(\gamma)$ the fund the risk aversion of which is the closest to $\gamma$. We assume that the set of risk aversions $\gamma_{j}$ is such that the values $J(\gamma)$ for $\gamma$ used below are unique.

Theorem 6.1 The stock price satisfies the SDE

$$
S_{t}^{-1} d S_{t}=\mu_{t} d t+\sigma_{t} d B_{t}
$$

where, for any $\lambda \in \Pi_{j}$,

$$
\lim _{T \rightarrow \infty} \sigma_{t}=\sigma\left(1+\gamma_{J\left(1+\gamma_{j}\right)}-\gamma_{0}\right)
$$

and

$$
\lim _{T \rightarrow \infty} \mu_{t}=r+\gamma_{J\left(1+\gamma_{j}\right)}\left(1+\gamma_{J\left(1+\gamma_{j}\right)}-\gamma_{0}\right) \sigma^{2}
$$


Remark 6.1 By Theorem 5.1, the behavior of the stock price $S_{t}$ changes drastically as $\lambda$ crosses the boundary of one of the intervals $\Pi_{j}$. The drift of $S_{t}$ may depend on the behavior of $f_{i}\left(t \lambda^{-1}\right)$, but (8) suggests that perhaps the volatility $\sigma_{t}$ is given by $\sigma\left(1-\gamma_{0}+\gamma_{j}\right)$ for $\lambda \in \Pi_{j}$. Equation (9) suggests that for $\lambda \in \Pi_{0}$, i.e., the interval closest to the horizon $T$, we should have $\mu_{t} \sim \gamma_{0} \sigma^{2}, \sigma_{t} \sim \sigma$. Theorem 6.1 shows that this is not true! By the definition of $J\left(1+\gamma_{j}\right)$, we always have $\gamma_{J\left(1+\gamma_{j}\right)} \geq \gamma_{j}$ and therefore

$$
\lim _{T \rightarrow \infty} \sigma_{t} \geq \sigma\left(1-\gamma_{0}+\gamma_{j}\right)
$$

and, for $\lambda \in \Pi_{0}$,

$$
\lim _{T \rightarrow \infty} \mu_{t} \geq \gamma_{0} \sigma^{2}
$$

Let, for example, $\gamma_{0}=1$ and $\gamma_{J(2)}=2$. Then, for $\lambda \in \Pi_{0}$, we get $\sigma_{t} \rightarrow 2 \sigma$ and $\mu_{t} \rightarrow 4 \sigma^{2}$. Thus, even though the price in that interval corresponds to a market with only the log fund present, as shown in Theorem 5.1, its drift and volatility are affected also by the risk aversion closest to 2. In essence, there is a delayed effect by a more risk averse fund on the mean return rate and the volatility.

KRWW (2006) also find that the price volatility may differ from the one conjectured from the asymptotic behavior of the stock price. However, KRWW (2006) show that, for $\lambda$ sufficiently close to one, both drift and volatility are determined solely by the surviving agent. Our Theorem 6.1 implies that non-surviving agents can generate impact on drift and volatility for all $\lambda \in(0,1)$. This is a new and surprising phenomenon.

The following result is a direct consequence of Theorem 6.1 .

Corollary 6.1 In the limit $T \rightarrow \infty$, the instantaneous drift, the volatility and the Sharpe ratio of the stock are monotone decreasing in $t=\lambda T$.

By Proposition 6.4, the size of drift and volatility are determined by the representative agent's risk aversion $\gamma^{U}(x)$ of the economy. As time goes by, the contribution to $\gamma^{U}$ of the agents with large risk aversion becomes smaller (Theorem 5.1 indicates that their price impact vanishes gradually) and the average risk aversion should decrease and drive the stock characteristics down.

Next, also by Proposition 6.4, it is clear that for getting results on the portfolio weights we must understand the asymptotic behavior of $\gamma^{U}(x)$. The following non-trivial lemma is crucial for the proof of Theorem 6.1.

Lemma 6.2 Let either $t=\lambda T$ for some $\lambda \in[0,1)$ or $t$ be fixed. Then,

$$
\lim _{T \rightarrow \infty} \frac{E_{t}\left[\gamma^{U}(D) D^{\alpha}\right]}{E_{t}\left[D^{\alpha}\right]}=\gamma_{J(1-\alpha)} .
$$


Lemma 6.2 shows that any risk aversion in the economy can be generated by appropriately choosing $\alpha$. For example, for $\alpha=0$, we get the very intuitive result:

$$
\lim _{T \rightarrow \infty} E_{t}\left[\gamma^{U}(D)\right]=\gamma_{0}
$$

As agent 0 dominates in the long run, the average risk aversion of the economy converges to $\gamma_{0}$. However, for $\alpha$ sufficiently different from 0 we may get a very different behavior. Using Theorem 5.1 and Lemma 6.2, it is possible to prove

Proposition 6.5 (1) For any $\lambda \in(0,1]$, we have

$$
\lim _{T \rightarrow \infty} \frac{E_{t}\left[M D \gamma^{U}(D)\right]}{E_{t}[M D]}=\gamma_{0}
$$

(2) For any $\lambda \in \Pi_{j}$,

$$
\lim _{T \rightarrow \infty} \frac{E_{t}\left[M \gamma^{U}(D)\right]}{E_{t}[M]}=\gamma_{J\left(1+\gamma_{j}\right)}
$$

See Appendix for details. Theorem 6.1 is a direct consequence of Propositions 6.4 and 6.5.

\subsection{Limit portfolios}

Let $i>0, \gamma_{i}>1$, and define the intervals $\Theta_{0}^{i}, \cdots, \Theta_{i-1}^{i}$ as follows: we set

$$
\Theta_{0}^{i}=\left(\frac{2-\left(\gamma_{0}+\gamma_{1}\right) b_{i}}{\left(\gamma_{0}+\gamma_{1}\right)\left(1-b_{i}\right)}, 1\right)
$$

and, for $j \in\{1, \ldots, i-2\}$,

$$
\Theta_{j}^{i}=\left(\frac{2-\left(\gamma_{j}+\gamma_{j+1}\right) b_{i}}{\left(\gamma_{j}+\gamma_{j+1}\right)\left(1-b_{i}\right)}, \frac{2-\left(\gamma_{j-1}+\gamma_{j}\right) b_{i}}{\left(\gamma_{j-1}+\gamma_{j}\right)\left(1-b_{i}\right)}\right)
$$

and, finally,

$$
\Theta_{i-1}^{i}=\left(0, \frac{2-\left(\gamma_{i-1}+\gamma_{i}\right) b_{i}}{\left(\gamma_{i-1}+\gamma_{i}\right)\left(1-b_{i}\right)}\right) .
$$

We start with the following

Lemma 6.3 Fix an $i$ such that $\gamma_{i} \geq 1$. Then, for any $\lambda \in \Theta_{j}^{i}$,

$$
\lim _{T \rightarrow \infty} \frac{E_{t}\left[M^{1-b_{i}} \gamma^{U}(D)\right]}{E_{t}\left[M^{1-b_{i}}\right]}=\gamma_{J\left(1+\gamma_{j}\left(1-b_{i}\right)\right)} .
$$

Proof: Directly from Lemmas 6.2 and D.3 in Appendix.

The main result in the subsection is the following limiting behavior of the portfolio weights of the funds more risk averse than log: 
Theorem 6.2 Let $t=\lambda T$ and fix an $i$ such that $\gamma_{i} \geq 1$. Then, for any $\lambda \in \Theta_{j}^{i} \cap \Pi_{m}$,

$$
\lim _{T \rightarrow \infty} \pi_{i t}=\pi_{i}(j, m)=\frac{\left(b_{i}-1\right) \gamma_{J\left(1+\gamma_{j}\left(1-b_{i}\right)\right)}+\gamma_{J\left(1+\gamma_{m}\right)}}{1-\gamma_{0}+\gamma_{J\left(1+\gamma_{m}\right)}} .
$$

If $\gamma_{0} \geq 1$ then, for any $\lambda$ except, maybe, for a finite set of values,

$$
\lim _{T \rightarrow \infty} \pi_{0 t}=1
$$

Proof: Directly from Propositions 6.4, 6.5 and Lemma 6.3.

We can decompose the limit portfolio

$$
\pi_{i}(j, m)=\pi_{i}(j, m)^{\text {myopic }}+\pi_{i}(j, m)^{\text {hedging }}
$$

By Theorem 6.1, the myopic component of the limit portfolio is given by

$$
\pi_{i}(j, m)^{\text {myopic }}=\lim _{T \rightarrow \infty} \frac{\mu_{t}-r}{\gamma_{i} \sigma_{t}^{2}}=\frac{b_{i} \gamma_{J\left(1+\gamma_{m}\right)}}{1-\gamma_{0}+\gamma_{J\left(1+\gamma_{m}\right)}}
$$

and therefore, the hedging component is given by

$$
\pi_{i}(j, m)^{\text {hedging }}=\left(1-b_{i}\right) \frac{\gamma_{J\left(1+\gamma_{m}\right)}-\gamma_{J\left(1+\gamma_{j}\left(1-b_{i}\right)\right)}}{1-\gamma_{0}+\gamma_{J\left(1+\gamma_{m}\right)}} .
$$

We know by Proposition 6.2 that the hedging component is nonnegative. This can also be seen directly. In fact, a direct calculation shows that

$$
\frac{2-\left(\gamma_{j}+\gamma_{j+1}\right) b_{i}}{\left(\gamma_{j}+\gamma_{j+1}\right)\left(1-b_{i}\right)}<\frac{2}{\gamma_{j}+\gamma_{j+1}}
$$

Consequently, if the intersection $\Theta_{j}^{i} \cap \Pi_{m}$ is non-empty, then necessarily $\gamma_{j} \leq \gamma_{m}$ and therefore

$$
J\left(1+\gamma_{m}\right) \geq J\left(1+\gamma_{j}\right) \geq J\left(1+\gamma_{j}\left(1-b_{i}\right)\right),
$$

proving the hedging component is no less than zero. Actually, if the set of risk aversions in the economy is sufficiently dense, it is seen from the above formula that the hedging component will be strictly positive. In other words, the presence of agents with varying risk aversions induces hedging in the optimal portfolio strategies.

Note that (18) implies, in particular, $\Pi_{0} \subset \Theta_{0}^{i}$. We analyze this special and important case of $\lambda \in \Pi_{0}$ in a separate

Proposition 6.6 For $\lambda \in \Pi_{0}$,

$$
\pi_{i}(0,0)^{\text {hedging }}=\left(1-b_{i}\right) \frac{\gamma_{J\left(1+\gamma_{0}\right)}-\gamma_{J\left(1+\gamma_{0}\left(1-b_{i}\right)\right)}}{1-\gamma_{0}+\gamma_{J\left(1+\gamma_{0}\right)}} .
$$

In particular, if $J\left(1+\gamma_{0}\right)=J\left(1+\gamma_{0}\left(1-b_{i}\right)\right)$ then $\pi_{i}(0,0)^{\text {hedging }}=0$, otherwise it is strictly positive. 
For $\lambda \in(0,1)$ but not in $\Pi_{0}$, that is, for the intervals before the last, the presence of the hedging component is not surprising: the funds anticipate changes in the future investment opportunity set, in particular those when time reaches the last interval $\Pi_{0}$, and hedge against those future changes. KRWW (2006) were the first to discover this remarkable phenomenon: the hedging component may stay non-zero long after the price reaches a regime of constant drift and volatility (interval $\Pi_{0}$ in our setting). However, they also find that there is always a finite number $\tilde{\lambda}<1$ such that the hedging component vanishes for $\lambda>\tilde{\lambda}$. Proposition 6.6 shows that the behavior in our model with many agents may be different: either the hedging component is identically zero for all $\lambda \in \Pi_{0}$, or it is strictly positive for all $\lambda \in \Pi_{0}$. Even for the times arbitrarily close to $T$, agents may continue hedging against stochastic drift and volatility. As above, when the risk aversions of the agents cover a sufficiently dense set of values, there will be a positive hedging component in the optimal portfolio strategies in the very long run, although the price drift and volatility are close to being at its constant limit. This phenomenon is directly related to Theorems 5.1 and 6.1. Even for $\lambda \in \Pi_{0}$, the stock price still has a stochastic drift and volatility. Even though the drift and volatility converge to constant values asymptotically, there are small probability events on which they deviate from their limits and force the price itself to behave as if it had another drift and volatility, as indicated by Theorem 5.1. These small probability events are precisely the events on which non-surviving agents generate drift and volatility impact and force other agents to hedge against these events. ${ }^{10}$

On the other hand, for a sufficiently risk averse fund with $b_{i}$ close to zero, the hedging component will be close to zero in the very long run. It is the funds with lower risk aversions (but higher than one), which engage in substantial hedging in the long run.

For risk aversions less than log, we don't have the exact limit, but the following bounds for the portfolio weights:

Proposition 6.7 If $\gamma_{i}<1$, then for all $\lambda \in \Pi_{m}$,

$$
\begin{aligned}
\frac{\left(b_{i}-1\right) \gamma_{J\left(1+\gamma_{l}\left(1-b_{i}\right)\right)}+\gamma_{J\left(1+\gamma_{m}\right)}}{1-\gamma_{0}+\gamma_{J\left(1+\gamma_{m}\right)}} & \leq \lim \inf _{T \rightarrow \infty} \pi_{i t} \\
& \leq \lim \sup _{T \rightarrow \infty} \pi_{i t} \leq \frac{\left(b_{i}-1\right) \gamma_{J\left(1+\gamma_{-k}\left(1-b_{i}\right)\right)}+\gamma_{J\left(1+\gamma_{m}\right)}}{1-\gamma_{0}+\gamma_{J\left(1+\gamma_{m}\right)}} .
\end{aligned}
$$

The result follows from Proposition 6.4 and

Lemma 6.4 If $\alpha>0$ then

$$
\frac{E_{t}\left[D^{-\gamma_{-k} \alpha} \gamma^{U}(D)\right]}{E_{t}\left[D^{-\gamma_{-k} \alpha}\right]} \leq \frac{E_{t}\left[M^{\alpha} \gamma^{U}(D)\right]}{E_{t}\left[M^{\alpha}\right]} \leq \frac{E_{t}\left[D^{-\gamma_{l} \alpha} \gamma^{U}(D)\right]}{E_{t}\left[D^{-\gamma_{l} \alpha}\right]}
$$

and the inequalities reverse for $\alpha<0$.

\footnotetext{
${ }^{10}$ Such an impact on the state-price density by vanishing agents was present also in the model of KRWW (2006), where it was possible to compute it explicitly.
} 


\subsection{Continuous risk aversion limit}

The limit expressions in the previous section are difficult to digest because of their discrete nature. In particular, since the risk aversions take discrete values, the behavior of the function $\gamma_{J(\alpha)}$ becomes complicated. On the other hand, if the risk aversion is continuous, $\gamma_{J(\alpha)}=\alpha$ and many expressions start looking much cleaner. In this section we study the case when the risk aversions cover sufficiently densely an interval of the form $[1, \Gamma]$ for large enough $\Gamma$. In that case, intervals $\Pi_{i}$ become very small and $\lambda \in \Pi_{i}$ means that

$$
\lambda \approx \frac{2}{\gamma_{i}+\gamma_{i+1}} \approx \gamma_{i}^{-1} \Leftrightarrow \gamma_{i} \approx \lambda^{-1}
$$

As the risk aversions become more and more dense in the continuous risk aversion limit, this approximation becomes exact. We cannot make the distribution of risk aversion truly continuous, because then our convergence results do not hold any more, but when the risk aversions are sufficiently dense, the approximation errors become negligible ${ }^{11}$. Thus, we have the following analog of Corollary 5.1:

Corollary 6.2 In the continuous risk aversion limit, the long run per-period expected return is given, for $\lambda>\Gamma^{-1}$, by

$$
R(\lambda):=\lim _{T \rightarrow \infty}(T-t)^{-1} \log \left(\frac{E_{t}[D]}{S_{t}}\right)=\frac{1}{2} \sigma^{2}\left(1+\lambda^{-1}\right)+r .
$$

Since, in the continuous limit, $\gamma_{J\left(1+\gamma_{j}\right)}=1+\gamma_{j}$ and $\gamma_{j}=\lambda^{-1}$, Theorem 6.1 takes the form

Proposition 6.8 In the continuous risk aversion limit, for $\lambda>\Gamma^{-1}$,

$$
\sigma(\lambda):=\lim _{T \rightarrow \infty} \sigma_{\lambda T}=\sigma\left(1+\lambda^{-1}\right) \quad, \quad \mu(\lambda):=\lim _{T \rightarrow \infty} \mu_{\lambda T}=r+\left(1+\lambda^{-1}\right)^{2} \sigma^{2} .
$$

Similarly, in the continuous risk aversion limit, $\lambda \in \Theta_{j}^{i} \cap \Pi_{m}$ means that

$$
\lambda \approx \gamma_{j}^{-1}-\frac{1}{\gamma_{i}-1} \approx \gamma_{m}^{-1}
$$

that is

$$
\gamma_{j} \approx \frac{\gamma_{i}-1}{\lambda\left(\gamma_{i}-1\right)+1}, \gamma_{m} \approx \lambda^{-1}
$$

Thus, Theorem 6.2 takes the form

\footnotetext{
${ }^{11}$ It is also possible to prove our results directly for a continuum of agents when the risk aversion distribution is sufficiently smooth.
} 
Proposition 6.9 Let $\lambda>\Gamma^{-1}$ and

$$
\pi_{\gamma}(\lambda)=\lim _{T \rightarrow \infty} \pi_{\gamma, \lambda T}
$$

be the limit portfolio of the agent with risk aversion $\gamma$. Then, in the continuous risk aversion limit,

$$
\pi_{\gamma}(\lambda)=\frac{\lambda^{2} \gamma(\gamma-1)+\left(2\left(\gamma^{2}-\gamma\right)+1\right) \lambda+\gamma^{2}}{\gamma^{2}((\gamma-1) \lambda+1)(\lambda+1)}
$$

In particular,

$$
\pi_{\gamma}^{\text {myopic }}(\lambda)=\frac{1}{\gamma}
$$

is independent of $\lambda$ and

$$
\pi_{\gamma}^{\text {hedging }}(\lambda)=(\gamma-1) \frac{(\gamma-1) \lambda+\gamma}{\gamma^{2}((\gamma-1) \lambda+1)(\lambda+1)}
$$

is monotone decreasing in $\lambda$.

There are several surprising features here. The most interesting is that the formulae are independent of the risk aversion distribution. It does not matter what the cross-sectional distribution of risk aversion in the population is, it only matters that it covers all possible values sufficiently densely. Furthermore, we see that in that case the hedging component is monotone decreasing with time. This has a clear economic meaning: the closer we are to the terminal horizon $T$, the less stochastic fluctuations are left in future, against which the agent needs to hedge. However, interestingly enough, the hedging demand never vanishes completely. More precisely, we have

$$
\pi_{\gamma}^{\text {hedging }}\left(\Gamma^{-1}\right)>\pi_{\gamma}^{\text {hedging }}(\lambda)>\pi_{\gamma}^{\text {hedging }}(1)=\frac{2 \gamma^{2}-3 \gamma+1}{2 \gamma^{3}} .
$$

At the very beginning, when $\lambda$ is small, the agent expects a lot of fluctuations in the stochastic investment opportunity set and invests a large fraction into the hedging portfolio, that may approach one as his risk aversion $\gamma$ becomes sufficiently large. In particular, for sufficiently large $\Gamma$,

$$
\pi_{\gamma}\left(\Gamma^{-1}\right) \approx 1-\frac{1}{\gamma}
$$

is monotone increasing in risk aversion.

On the other hand, as time approaches $T$, the hedging demand decreases. A direct calculation shows that $\pi_{\gamma}^{\text {hedging }}(1)$ is monotone increasing on $\gamma \in[0,(3+\sqrt{3}) / 2]$ and decreasing for larger values. Thus, we discover the following unexpected phenomenon: for time horizons sufficiently close to $T$ and large enough risk aversion the hedging demand is monotone decreasing in risk aversion. 


\subsection{The case of two funds}

In this section we consider the case of an economy populated by two funds with risk aversions $\gamma_{1}>\gamma_{0}>1$. In this case,

$$
\Pi_{0}=\left(\frac{2}{\gamma_{0}+\gamma_{1}}, 1\right) \quad, \quad \Pi_{1}=\left(0, \frac{2}{\gamma_{0}+\gamma_{1}}\right) .
$$

For agent 1, we need to consider the intervals

$$
\Theta_{0}^{1}=\left(\frac{\gamma_{1}-\gamma_{0}}{\left(\gamma_{0}+\gamma_{1}\right)\left(\gamma_{1}-1\right)}, 1\right) \quad, \quad \Theta_{1}^{1}=\left(0, \frac{\gamma_{1}-\gamma_{0}}{\left(\gamma_{0}+\gamma_{1}\right)\left(\gamma_{1}-1\right)}\right) .
$$

Then, the interval $(0,1)$ will be partitioned into three intervals

$$
\Lambda_{1}=\Theta_{1}^{1}, \Lambda_{2}=\Theta_{0}^{1} \cap \Pi_{1}, \Lambda_{3}=\Pi_{0}=\left(\frac{2}{\gamma_{0}+\gamma_{1}}, 1\right)
$$

given by

$$
\left(0, \frac{\gamma_{1}-\gamma_{0}}{\left(\gamma_{0}+\gamma_{1}\right)\left(\gamma_{1}-1\right)}\right),\left(\frac{\gamma_{1}-\gamma_{0}}{\left(\gamma_{0}+\gamma_{1}\right)\left(\gamma_{1}-1\right)}, \frac{2}{\gamma_{0}+\gamma_{1}}\right),\left(\frac{2}{\gamma_{0}+\gamma_{1}}, 1\right)
$$

Let

$$
\left(\pi_{\gamma_{0}}^{\text {hedging }}(\lambda), \pi_{\gamma_{1}}^{\text {hedging }}(\lambda)\right)=\left(\lim _{T \rightarrow \infty} \pi_{\gamma_{0}, \lambda T}^{\text {hedging }}, \lim _{T \rightarrow \infty} \pi_{\gamma_{1}, \lambda T}^{\text {hedging }}\right)
$$

be the two-dimensional vector of the limit hedging components of the portfolios of agents 0 and 1 . We summarize our findings in

Proposition 6.10 We have

(1) If $\gamma_{1}<2$ then the long run drift and volatility

$$
\lim _{T \rightarrow \infty} \sigma_{t}=\sigma\left(1+\gamma_{1}-\gamma_{0}\right), \lim _{T \rightarrow \infty} \mu_{t}=r+\gamma_{1}\left(1+\gamma_{1}-\gamma_{0}\right) \sigma^{2}
$$

as well as the hedging components of the portfolios

$$
\left(\pi_{\gamma_{0}}^{\text {hedging }}(t), \pi_{\gamma_{1}}^{\text {hedging }}(t)\right)=\frac{\gamma_{1}-\gamma_{0}}{1+\gamma_{1}-\gamma_{0}}\left(\left(1-b_{0}\right), 0\right)
$$

are independent of $\lambda$.

(2) If $2<\gamma_{1}<2+\gamma_{0}$ then the long run drift and volatility are given by (19), but the hedging components are given by

$$
\left(\pi_{\gamma_{0}}^{\text {hedging }}(t), \pi_{\gamma_{1}}^{\text {hedging }}\right)=\frac{\gamma_{1}-\gamma_{0}}{1+\gamma_{1}-\gamma_{0}} \begin{cases}\left(\left(1-b_{0}\right), 0\right) & \lambda \in \Lambda_{1} ; \\ \left(\left(1-b_{0}\right),\left(1-b_{1}\right)\right) & \lambda \in \Lambda_{2} \cup \Lambda_{3} .\end{cases}
$$


(3) If $\gamma_{1}>2+\gamma_{0}$ then the long-run drift and volatility are given by

$$
\lim _{T \rightarrow \infty}\left(\sigma_{t}, \mu_{t}-r\right)= \begin{cases}\left(1+\gamma_{1}-\gamma_{0}\right)\left(\sigma, \sigma^{2} \gamma_{1}\right) & \lambda \in \Lambda_{1} \cup \Lambda_{2} ; \\ \left(\sigma, \sigma^{2} \gamma_{0}\right) & \lambda \in \Lambda_{3},\end{cases}
$$

and the hedging components of the portfolios are given by

$$
\left(\pi_{\gamma_{0}}^{\text {hedging }}(t), \pi_{\gamma_{1}}^{\text {hedging }}(t)\right)=\frac{\gamma_{1}-\gamma_{0}}{1+\gamma_{1}-\gamma_{0}} \begin{cases}\left(\left(1-b_{0}\right), 0\right) & \lambda \in \Lambda_{1} ; \\ \left(0,\left(1-b_{1}\right)\right) & \lambda \in \Lambda_{2} \\ (0,0) & \lambda \in \Lambda_{3} .\end{cases}
$$

Because of the sparse distribution of risk aversion (only two values), there are several phenomena with no analogs in the continuous risk aversion setting of the previous section. For example, the hedging portfolio of agent 0 is always decreasing in $\lambda$. But, the hedging portfolio of agent 1 is increasing in $\lambda$ in the case (2) and has a "kink" in case (3). The structure of the hedging portfolio appearing in case (3) is similar to those appearing in KRWW (2006). However, the structure arising in cases (1) and (2) is new.

In case (1), agent 1 chooses a time-independent, myopic portfolio, but the less risk averse agent 0 chooses a substantial hedging portfolio, even though the asymptotic drift and volatility are independent of $\lambda$. The reason is that the drift and volatility may deviate from their limit values and, by Theorem 5.1, for $\lambda \in \Pi_{0}=\Lambda_{3}$, the stock price behaves asymptotically as if it had a drift and volatility $\left(\sigma, r+\gamma_{0} \sigma^{2}\right)$. A positive hedging component independent of $\lambda$ is new and differs from KRWW (2006).

In case (2), the asymptotic drift and volatility are still time-independent and agent 0 holds the same portfolio. On the other hand, for small $\lambda$, when there are lot of possible fluctuations in the future investment opportunity set, agent 1 nevertheless decides not to hedge at all. However, for $\lambda \in \Pi_{0}$ (i.e., $\left.\lambda>2 /\left(\gamma_{0}+\gamma_{1}\right)\right)$, he chooses a non-trivial hedging portfolio. This monotone increasing structure of the hedging portfolio is different from the one discovered by KRWW (2006).

Finally, in case (3), agent 0 does not hedge for $\lambda \in \Lambda_{2} \cup \Lambda_{3}$, whereas agent 1 does not hedge at the beginning, hedges in the middle, and then again stops hedging.

\section{Conclusions}

We show that, with long-lived funds, the equilibrium in complete market models lead to Merton-Black-Scholes (MBS) type prices and portfolio strategies, asymptotically. However, the price parameters change as time goes by, leading to asymptotically lower and lower returns, and the portfolio weights are not myopic as in the partial equilibrium MBS model. 
The size of the price volatility is influenced by the overall dividend volatility and the difference between the highest and the lowest risk aversion. Eventually, the fund closest to the log preferences is the only one that survives, but price impact is felt for a long time also from other funds, more risk averse than log. The drift and the volatility of the price, as well as the optimal portfolio weights, depend on risk aversions higher than one even at the times closest to the final time horizon. The portfolios include a non-zero hedging component, even in the very long run. The hedging component is positive for the risk aversions higher than one, and negative for those smaller than one, and it may be monotone decreasing in risk aversion at further away times. In terms of the expected value of the relative wealth size, the log fund will not dominate even in the long run.

It would be of significant interest to show how much our results remain valid when there is also intermediate consumption of the funds. The optimal final consumption will still have the same form, so it is possible that some results will qualitatively survive. Moreover, it would be desirable to see what happens to the price and portfolio behavior in the presence of more than one risky asset, and with funds having different time horizons. The picture then would likely be more dynamic, with funds and/or assets entering and leaving the market as time goes by. Finally, once the behavior for standard rational agents is well understood, one can see how the equilibrium differs with non-standard preferences and behavior. In particular, in our model leveraging is low, as the funds' wealth needs to stay positive, and the probability of bankruptcy is zero. We leave the possibility of extending our analysis to these interesting issues for future research.

\section{Appendix}

\section{A Proofs for Section 3}

We will do the proofs of this section under a strengthened form of Assumption 3.1. Throughout this section we assume a power rate of convergence for the risk aversions:

Assumption A.1 There exist constants $K, a>0$ such that

$$
\left|\gamma_{i}(x)-\gamma_{i}^{0}\right| \leq K x^{a} \text { and }\left|\gamma_{i}(x)-\gamma_{i}^{\infty}\right| \leq K x^{-a}
$$

for all $i$.

Condition (21) is not necessary, but the proofs are much simpler than under Assumption 3.1. 
Lemma A.1 Under Assumption A.1, the limits

$$
\lim _{x \downarrow 0} \frac{u_{i}^{\prime}(x)}{x^{-\gamma_{i}^{0}}} \text { and } \lim _{x \rightarrow \infty} \frac{u_{i}^{\prime}(x)}{x^{-\gamma_{i}^{\infty}}}
$$

exist and are strictly positive for each $i$. Consequently, there exists a constant $K$ such that

$$
K^{-1}\left(\chi_{x>1} x^{-\gamma_{i}^{\infty}}+\chi_{x \leq 1} x^{-\gamma_{i}^{0}}\right) \leq u_{i}^{\prime}(x) \leq K\left(\chi_{x>1} x^{-\gamma_{i}^{\infty}}+\chi_{x \leq 1} x^{-\gamma_{i}^{0}}\right)
$$

and

$$
K^{-1}\left(\chi_{x>1} x^{-b_{i}^{0}}+\chi_{x \leq 1} x^{-b_{i}^{\infty}}\right) \leq I_{i}(x) \leq K\left(\chi_{x>1} x^{-b_{i}^{0}}+\chi_{x \leq 1} x^{-b_{i}^{\infty}}\right) .
$$

Here,

$$
b_{i}^{0}=\left(\gamma_{i}^{0}\right)^{-1}, b_{i}^{\infty}=\left(\gamma_{i}^{\infty}\right)^{-1} .
$$

Proof of Lemma A.1: Under the normalization $u_{i}^{\prime}(1)=1$,

$$
\log \left(u_{i}^{\prime}(x) x^{\gamma_{i}^{0}}\right)=\int_{x}^{1} \gamma_{i}(s) s^{-1} d s-\gamma_{i}^{0} \int_{x}^{1} s^{-1} d s=\int_{x}^{1}\left(\gamma_{i}(s)-\gamma_{i}^{0}\right) s^{-1} d s .
$$

Since $\left|\gamma_{i}(s)-\gamma_{i}^{0}\right|<K s^{a}$, the singularity is integrable and we get that

$$
\lim _{x \downarrow 0} \int_{x}^{1}\left(\gamma_{i}(s)-\gamma_{i}^{0}\right) s^{-1} d s=\int_{0}^{1}\left(\gamma_{i}(s)-\gamma_{i}^{0}\right) s^{-1} d s=L
$$

is finite and

$$
\lim _{x \downarrow 0} \frac{u_{i}^{\prime}(x)}{x^{-\gamma_{i}^{0}}}=e^{L}
$$

The case $x \rightarrow \infty$ is analogous.

Lemma A.2 Let $M$ be an arbitrary, positive random variable. Let also

$$
W_{i T}=I_{i}\left(\lambda_{i} M\right), E\left[M I_{i}\left(\lambda_{i} M\right)\right]=E\left[\psi_{i} D M\right]
$$

If $\gamma_{i}^{0}, \gamma_{i}^{\infty}>1$, then there exists a constant $K>0$, independent of $T$ and $M, D$, such that

$$
E\left[W_{i T} u_{i}^{\prime}\left(W_{i T}\right)\right] \leq K E\left[D u_{i}^{\prime}(D)\right]
$$

If $\gamma_{i}^{0}, \gamma_{i}^{\infty}<1$, then there exists a constant $K>0$, independent of $T$ and $M, D$, such that

$$
E\left[W_{i T} u_{i}^{\prime}\left(W_{i T}\right) \geq K E\left[D u_{i}^{\prime}(D)\right]\right.
$$

Proof of Lemma A.2: By Lemma A.1, it is sufficient to consider the case

$$
u_{i}^{\prime}(x)=\chi_{x>1} x^{-\gamma_{i}^{\infty}}+\chi_{x \leq 1} x^{-\gamma_{i}^{0}}
$$


and $^{12}$

$$
I_{i}(x)=\chi_{x>1} x^{-b_{i}^{0}}+\chi_{x \leq 1} x^{-b_{i}^{\infty}} .
$$

We only prove the case $\gamma_{i}^{0}, \gamma_{i}^{\infty}>1$, the other case being analogous.

If $\gamma_{i}^{\infty} \leq \gamma_{i}^{0}$ then the function

$$
x u_{i}^{\prime}(x)=\chi_{x>1} x^{1-\gamma_{i}^{\infty}}+\chi_{x \leq 1} x^{1-\gamma_{i}^{0}}
$$

is convex and therefore the solution to the minimization problem

$$
\min \left\{E\left[X u_{i}^{\prime}(X)\right]: E[M X]=E\left[\psi_{i} D M\right]\right\}
$$

is given by

$$
\hat{X}=f_{i}^{-1}\left(\rho_{i} M\right)
$$

where

$$
f_{i}(x)=\left(\gamma_{i}^{\infty}-1\right) \chi_{x>1} x^{-\gamma_{i}^{\infty}}+\left(\gamma_{i}^{0}-1\right) \chi_{x \leq 1} x^{-\gamma_{i}^{0}}
$$

and $\rho_{i}$ is the Lagrange multiplier.

If $\gamma_{i}^{\infty}>\gamma_{i}^{0}$, we take a convex modification of $x u_{i}^{\prime}(x)$ by considering the function, also denoted $u_{i}^{\prime}$, with a slight abuse of notation,

$$
u_{i}^{\prime}(x)=\chi_{x>1} x^{-\gamma_{i}^{\infty}}+\chi_{a \leq x \leq 1}\left((1-x)\left(\gamma_{i}^{\infty}-1\right)+1\right)+\chi_{0<x<a} x^{-\gamma_{i}^{0}}
$$

with $a$ being the unique solution to

$$
(1-a)\left(\gamma_{i}^{\infty}-1\right)+1=a^{-\gamma_{i}^{0}}
$$

Then, $x u_{i}^{\prime}(x)$ is convex and the optimum is again attained at

$$
\hat{X}=\left(\left(x u_{i}^{\prime}(x)\right)^{\prime}\right)^{-1}\left(\rho_{i} M\right) \text {. }
$$

Because only the asymptotic behavior at zero and infinity matter, it is sufficient to consider again

$$
\hat{X}=f_{i}^{-1}\left(\rho_{i} M\right) \text {. }
$$

More precisely, for such $\hat{X}$, there is a constant $K$ such that

$$
E\left[\hat{X} u_{i}^{\prime}(\hat{X})\right] \leq K \min \left\{E\left[X u_{i}^{\prime}(X)\right]: E[M X]=E\left[\psi_{i} D M\right]\right\} .
$$

Since $X=\psi_{i} D$ obviously satisfies the budget constraint $E[M X]=E\left[\psi_{i} D M\right]$, and because, for some $K>0$,

$$
\psi_{i} D u_{i}^{\prime}\left(\psi_{i} D\right) \leq K \max \left\{\psi_{i}^{-\gamma_{i}^{0}}, \psi_{i}^{-\gamma_{i}^{\infty}}\right\} D u_{i}^{\prime}(D),
$$

\footnotetext{
${ }^{12}$ The function $I_{i}$ is not the inverse of $u_{i}^{\prime}$, but it has the same asymptotic behavior, which is enough for the estimates.
} 
we get

$$
E\left[\hat{X} u_{i}^{\prime}(\hat{X})\right] \leq K \min \left\{E\left[X u_{i}^{\prime}(X)\right]: E[M X]=E\left[\psi_{i} D M\right]\right\} \leq K E\left[D u_{i}^{\prime}(D)\right]
$$

Now, by definition, $\rho_{i}$ and $\lambda_{i}$ satisfy

$$
E\left[M I_{i}\left(\lambda_{i} M\right)\right]=E\left[M f_{i}^{-1}\left(\rho_{i} M\right)\right]=E\left[\psi_{i} D M\right]
$$

and by Lemma A.1,

$$
K_{1} f_{i}^{-1}(x) \leq I_{i}(x) \leq K_{2} f_{i}^{-1}(x)
$$

Therefore, we obtain

$$
K_{1} E\left[M f_{i}^{-1}\left(\lambda_{i} M\right)\right] \leq E\left[M f_{i}^{-1}\left(\rho_{i} M\right)\right] \leq K_{2} E\left[M f_{i}^{-1}\left(\lambda_{i} M\right)\right]
$$

Obviously, changing the constants $K_{1}$ and $K_{2}$, we may replace $f_{i}^{-1}$ in these inequalities by

$$
\chi_{x>1} x^{-b_{i}^{0}}+\chi_{x \leq 1} x^{-b_{i}^{\infty}} .
$$

Let

$$
L_{2}>\max \left\{K_{2}^{\gamma_{i}^{0}}, K_{2}^{\gamma_{i}^{\infty}}\right\}
$$

Suppose that

$$
\lambda_{i}>L_{2} \rho_{i} .
$$

Then,

$$
f_{i}^{-1}\left(\lambda_{i} M\right)<\max \left\{L_{2}^{-b_{i}^{0}}, L_{2}^{-b_{i}^{\infty}}\right\} f_{i}^{-1}\left(\rho_{i} M\right)<K_{2}^{-1} f_{i}^{-1}\left(\rho_{i} M\right)
$$

which contradicts (22). Similarly, choosing

$$
L_{1}=\min \left\{K_{1}^{\gamma_{i}^{0}}, K_{1}^{\gamma_{i}^{\infty}}\right\}
$$

we get that

$$
L_{1} \leq \lambda_{i} / \rho_{i} \leq L_{2}
$$

for constants $L_{1}, L_{2}>0$, independent of $T$ and $D$, and therefore Lemma A.1 implies that

$$
K_{1} f_{i}^{-1}\left(\rho_{i} M\right) \leq W_{i T}=I_{i}\left(\lambda_{i} M\right) \leq K_{2} f_{i}^{-1}\left(\rho_{i} M\right)
$$

for some $K_{1}, K_{2}>0$ and the required assertion follows from

$$
E\left[W_{i T} u_{i}^{\prime}\left(W_{i T}\right)\right] \leq K_{1} E\left[X u_{i}^{\prime}(X)\right] \leq K_{2} E\left[D u_{i}^{\prime}(D)\right]
$$

for some $K_{1}, K_{2}>0$. 
Lemma A.3 Assume Assumptions A.1 and 3.2. Then, there exist constants $K_{1}, K_{2}>0$, independent of $T$ and $D$ such that, in equilibrium,

$$
K_{1} \leq \frac{E\left[W_{i T} u_{i}^{\prime}\left(W_{i T}\right)\right]}{E\left[D u_{i}^{\prime}(D)\right]} \leq K_{2}
$$

Proof: By Lemma A.1, we may replace $u_{i}^{\prime}$ by

$$
\chi_{x>1} x^{-\gamma_{i}^{\infty}}+\chi_{x \leq 1} x^{-\gamma_{i}^{0}} .
$$

Then, the case $\gamma_{i}^{0}=\gamma_{i}^{\infty}=1$ is trivial. Let $\gamma_{i}^{0}, \gamma_{i}^{\infty}>1$. In equilibrium, $W_{i T} \leq D$, and therefore, since

$$
g_{i}(x)=\chi_{x>1} x^{1-\gamma_{i}^{\infty}}+\chi_{x \leq 1} x^{1-\gamma_{i}^{0}}
$$

is monotone decreasing, we get

$$
g_{i}\left(W_{i T}\right) \geq g_{i}(D)
$$

and Lemma A.1 implies

$$
E\left[W_{i T} u_{i}^{\prime}\left(W_{i T}\right)\right] \geq K_{1} E\left[D u_{i}^{\prime}(D)\right]
$$

for some $K_{1}>0$. The upper bound is contained in Lemma A.2. The case $\gamma_{i}^{0}, \gamma_{i}^{\infty}<1$ is completely analogous.

We are now ready to prove an estimate for the "wealth scaling" $\lambda_{i}$.

Lemma A.4 Assume Assumptions A.1 and 3.2. Then, there exist constants $K_{1}, K_{2}>0$ such that

$$
K_{1} \frac{E\left[D u_{i}^{\prime}(D)\right]}{E[M D]} \leq \lambda_{i} \leq K_{2} \frac{E\left[D u_{i}^{\prime}(D)\right]}{E[M D]}
$$

Proof: We have

$$
E\left[W_{i T} u_{i}^{\prime}\left(W_{i T}\right)\right]=E\left[I_{i}\left(\lambda_{i} M\right) u_{i}^{\prime}\left(I_{i}\left(\lambda_{i} M\right)\right)\right]=\lambda_{i}\left[M I_{i}\left(\lambda_{i} M\right)\right]=\lambda_{i} \psi_{i} E[D M]
$$

and the required estimate follows from Lemma A.3.

Finally, we can prove the following

Lemma A.5 Assume Assumptions A.1 and 3.2. Then, there exists a constant $K>0$ such that, for any two funds $i, j$,

$$
W_{i T} \leq K I_{i}\left(\frac{E\left[D u_{i}^{\prime}(D)\right]}{E\left[D u_{j}^{\prime}(D)\right]} u_{j}^{\prime}(D)\right) .
$$


Proof: We have

$$
M \geq \lambda_{j}^{-1} u_{j}^{\prime}(D)
$$

and therefore, since $I_{i}(x)$ is decreasing,

$$
W_{i T}=I_{i}\left(\lambda_{i} M\right) \leq I_{i}\left(\lambda_{i} \lambda_{j}^{-1} u_{j}^{\prime}(D)\right)
$$

and the claim follows from Lemma A.4.

The following lemma is straightforward.

Lemma A.6 Let

$$
N(x)=\frac{1}{\sqrt{2 \pi}} \int_{-\infty}^{x} e^{-t^{2} / 2} d t
$$

Then,

$$
\lim _{x \rightarrow-\infty} \frac{N(x)}{e^{-x^{2} / 2}(-x)^{-1}}=\lim _{x \rightarrow+\infty} \frac{1-N(x)}{e^{-x^{2}} x^{-1}}=\frac{1}{\sqrt{2 \pi}}
$$

We are now ready for the proof of the theorem.

Proof of Theorem 3.1: As usual, we only consider the case $\gamma_{0}^{0}, \gamma_{0}^{\infty}>1$. By definition of fund 0 , we have

$$
\min \left\{\gamma_{i}^{0}, \gamma_{i}^{\infty}\right\}>\max \left\{\gamma_{0}^{0}, \gamma_{0}^{\infty}\right\}
$$

We will use the bound (23) and show that its right-hand side, divided by $D$, converges to zero as $T \rightarrow \infty$. As before, for the asymptotic behavior of $E\left[D u_{0}^{\prime}(D)\right]$, it is sufficient to consider

$$
\begin{aligned}
E\left[D \left(\chi_{D>1} D^{-\gamma_{0}^{\infty}}+\right.\right. & \left.\left.\chi_{D \leq 1} D^{-\gamma_{0}^{0}}\right)\right] \\
& =K \sqrt{2 \pi}^{-1} T^{-1 / 2}\left(\int_{-\rho \sigma^{-1} T}^{+\infty} e^{(\rho T+\sigma x)\left(1-\gamma_{0}^{\infty}\right)} e^{-x^{2} /(2 T)} d x\right. \\
& \left.+\int_{-\infty}^{-\rho \sigma^{-1} T} e^{(\rho T+\sigma x)\left(1-\gamma_{0}^{0}\right)} e^{-x^{2} /(2 T)} d x\right) .
\end{aligned}
$$

Now, changing the variables we get

$$
\begin{aligned}
T^{-1 / 2} \int_{-\rho \sigma^{-1} T}^{+\infty} e^{(\rho T+\sigma x)\left(1-\gamma_{0}^{\infty}\right)} e^{-x^{2} /(2 T)} d x & \\
& =e^{\rho T \gamma_{0}^{\infty}} \int_{-\rho \sigma^{-1} T^{1 / 2}}^{+\infty} e^{\left(\sigma T^{1 / 2} x\right)\left(1-\gamma_{0}^{\infty}\right)} e^{-x^{2} / 2} d x \\
& =e^{\rho T\left(1-\gamma_{0}^{\infty}\right)} e^{\left(\sigma T^{1 / 2}\left(1-\gamma_{0}^{\infty}\right)\right)^{2} / 2} \int_{-\rho \sigma^{-1} T^{1 / 2}-\left(\sigma T^{1 / 2}\right)\left(1-\gamma_{0}^{\infty}\right)}^{+\infty} e^{-x^{2} / 2} d x
\end{aligned}
$$


Similarly,

$$
\begin{aligned}
T^{-1 / 2} \int_{-\infty}^{-\rho \sigma^{-1} T} e^{(\rho T+\sigma x)\left(1-\gamma_{0}^{0}\right)} e^{-x^{2} /(2 T)} d x & \\
& =e^{\rho T \gamma_{0}^{0}} \int_{-\rho \sigma^{-1} T^{1 / 2}}^{+\infty} e^{\left(\sigma T^{1 / 2} x\right)\left(1-\gamma_{0}^{0}\right)} e^{-x^{2} / 2} d x \\
& =e^{\rho T\left(1-\gamma_{0}^{0}\right)} e^{\left(\sigma T^{1 / 2}\left(1-\gamma_{0}^{0}\right)\right)^{2} / 2} \int_{-\infty}^{-\rho \sigma^{-1} T^{1 / 2}-\left(\sigma T^{1 / 2}\right)\left(1-\gamma_{0}^{0}\right)} e^{-x^{2} / 2} d x .
\end{aligned}
$$

Thus, the asymptotic behavior of $E\left[D u_{0}^{\prime}(D)\right]$ coincides with that of

$$
\begin{aligned}
e^{T\left(\rho\left(1-\gamma_{0}^{0}\right)+\left(\sigma\left(1-\gamma_{0}^{0}\right)\right)^{2} / 2\right)} & N\left(-\sqrt{T}\left(\rho \sigma^{-1}+\sigma\left(1-\gamma_{0}^{0}\right)\right)\right) \\
& +e^{T\left(\rho\left(1-\gamma_{0}^{\infty}\right)+\left(\sigma\left(1-\gamma_{0}^{\infty}\right)\right)^{2} / 2\right)}\left(1-N\left(-\sqrt{T}\left(\rho \sigma^{-1}+\sigma\left(1-\gamma_{0}^{\infty}\right)\right)\right)\right)
\end{aligned}
$$

and, similarly, for the case of $E\left[D u_{0}^{\prime}(D)\right]$. Suppose that the drift $\rho$ is sufficiently large, so that

$$
\rho \sigma^{-2} \geq \max \left\{\gamma_{i}^{\infty}-1, \gamma_{i}^{0}-1\right\}
$$

Then, by Lemma A.6,

$$
N\left(-\sqrt{T}\left(\rho \sigma^{-1}+\sigma\left(1-\gamma_{0}^{0}\right)\right)\right) \sim e^{-T\left(\rho \sigma^{-1}+\sigma\left(1-\gamma_{0}^{0}\right)\right)^{2} / 2}\left(\sqrt{T}\left(\rho \sigma^{-1}+\sigma\left(1-\gamma_{0}^{0}\right)\right)\right)^{-1}
$$

and hence

$$
\begin{aligned}
e^{T\left(\rho\left(1-\gamma_{0}^{0}\right)+\left(\sigma\left(1-\gamma_{0}^{0}\right)\right)^{2} / 2\right)} N\left(-\sqrt{T}\left(\rho \sigma^{-1}+\right.\right. & \left.\left.\sigma\left(1-\gamma_{0}^{0}\right)\right)\right) \\
& \sim e^{-T \rho^{2} \sigma^{-2} / 2}\left(\sqrt{T}\left(\rho \sigma^{-1}+\sigma\left(1-\gamma_{0}^{0}\right)\right)\right)^{-1} .
\end{aligned}
$$

We have

$$
\rho\left(1-\gamma_{0}^{\infty}\right)+\left(\sigma\left(1-\gamma_{0}^{\infty}\right)\right)^{2} / 2+\rho^{2} \sigma^{-2} / 2=\left(\rho \sigma^{-1}+\sigma\left(1-\gamma_{0}^{\infty}\right)\right)^{2}>0
$$

and therefore

$$
\begin{aligned}
& e^{T\left(\rho\left(1-\gamma_{0}^{0}\right)+\left(\sigma\left(1-\gamma_{0}^{0}\right)\right)^{2} / 2\right)} N\left(-\sqrt{T}\left(\rho \sigma^{-1}+\sigma\left(1-\gamma_{0}^{0}\right)\right)\right) \\
& +e^{T\left(\rho\left(1-\gamma_{0}^{\infty}\right)+\left(\sigma\left(1-\gamma_{0}^{\infty}\right)\right)^{2} / 2\right)}\left(1-N\left(-\sqrt{T}\left(\rho \sigma^{-1}+\sigma\left(1-\gamma_{0}^{\infty}\right)\right)\right)\right) \\
& \sim e^{T\left(\rho\left(1-\gamma_{0}^{\infty}\right)+\left(\sigma\left(1-\gamma_{0}^{\infty}\right)\right)^{2} / 2\right)}
\end{aligned}
$$

and, similarly,

$$
\begin{aligned}
& e^{T\left(\rho\left(1-\gamma_{i}^{0}\right)+\left(\sigma\left(1-\gamma_{0}^{0}\right)\right)^{2} / 2\right)} N\left(-\sqrt{T}\left(\rho \sigma^{-1}+\sigma\left(1-\gamma_{i}^{0}\right)\right)\right) \\
& +e^{T\left(\rho\left(1-\gamma_{i}^{\infty}\right)+\left(\sigma\left(1-\gamma_{i}^{\infty}\right)\right)^{2} / 2\right)}\left(1-N\left(-\sqrt{T}\left(\rho \sigma^{-1}+\sigma\left(1-\gamma_{i}^{\infty}\right)\right)\right)\right) \\
& \sim e^{T\left(\rho\left(1-\gamma_{i}^{\infty}\right)+\left(\sigma\left(1-\gamma_{i}^{\infty}\right)\right)^{2} / 2\right)} .
\end{aligned}
$$


Since, by assumption, $\rho>0, D$ converges to $+\infty$ almost surely by the law of large numbers and therefore

$$
u_{0}^{\prime}(D) \sim K D^{-\gamma_{0}^{\infty}} .
$$

Hence,

$$
\begin{aligned}
\frac{E\left[D u_{i}^{\prime}(D)\right]}{E\left[D u_{0}^{\prime}(D)\right]} u_{0}^{\prime}(D) \geq K_{1} \frac{e^{T\left(\rho\left(1-\gamma_{i}^{\infty}\right)+\left(\sigma\left(1-\gamma_{i}^{\infty}\right)\right)^{2} / 2\right)}}{e^{T\left(\rho\left(1-\gamma_{0}^{\infty}\right)+\left(\sigma\left(1-\gamma_{0}^{\infty}\right)\right)^{2} / 2\right)}} e^{-\rho \gamma_{0}^{\infty}} e^{-\sigma \gamma_{0}^{\infty} B_{T}} \\
=e^{-\rho \gamma_{i}^{\infty}+\sigma^{2}\left(\left(1-\gamma_{i}^{\infty}\right)^{2}-\left(1-\gamma_{i}^{0}\right)^{2}\right) / 2} e^{-\sigma \gamma_{0}^{\infty} B_{T}}
\end{aligned}
$$

Since, by assumption,

$$
\rho \sigma^{-2} \gamma_{i}^{\infty}>\left(\gamma_{i}^{\infty}-1\right) \gamma_{i}^{\infty}>\left(\gamma_{i}^{\infty}-1\right)^{2},
$$

the expression (32) converges to zero as $T \rightarrow \infty$ by the law of large numbers and therefore

$$
\begin{aligned}
I_{i}\left(\frac{E\left[D u_{i}^{\prime}(D)\right]}{E\left[D u_{0}^{\prime}(D)\right]} u_{0}^{\prime}(D)\right) D^{-1} \sim & K\left(\frac{E\left[D u_{i}^{\prime}(D)\right]}{E\left[D u_{0}^{\prime}(D)\right]} u_{0}^{\prime}(D)\right)^{-\left(\gamma_{i}^{\infty}\right)^{-1}} D^{-1} \\
& \leq K_{1} e^{-\sigma^{2}\left(\gamma_{i}^{\infty}\right)^{-1}\left(\left|1-\gamma_{i}^{\infty}\right|^{2}-\left|1-\gamma_{0}^{\infty}\right|^{2}\right)+\sigma\left(\gamma_{0}^{\infty}\left(\gamma_{i}^{\infty}\right)^{-1}-1\right) B_{T}}
\end{aligned}
$$

converges to zero by the law of large numbers.

The cases when $\rho$ does not satisfy (28) are similar, but require very lengthy calculations and we omit them here.

It follows that

$$
\frac{W_{i T}}{D} \rightarrow 0
$$

for all $i \neq 0$. By market clearing,

$$
\frac{W_{0 T}}{D}=1-\sum_{i \neq 0} \frac{W_{i T}}{D} \rightarrow 1
$$

and the claim follows.

\section{B Proofs for Section 4}

Lemma B.1 If $\gamma_{j}(x)>\gamma_{i}(y)$ for all $x, y$, then the function

$$
f(x)=\frac{I_{j}(a x)}{I_{i}(b x)}
$$

is monotone increasing for any $a, b>0$. In particular, if $\gamma_{j}(x) \geq 1$ for all $x$, then $x I_{j}(x)$ is monotone increasing, and if $\gamma_{j}(x) \leq 1$ for all $x$, then $x I_{j}(x)$ is monotone decreasing. 
Proof: By definition,

$$
u_{j}^{\prime}\left(I_{j}(x)\right)=x
$$

and therefore

$$
I_{j}^{\prime}(x)=u_{j}^{\prime \prime}\left(I_{j}(x)\right)^{-1} .
$$

Thus, we have, with $z_{1}=I_{j}(a x), z_{2}=I_{i}(b x)$,

$$
\begin{array}{r}
f^{\prime}(x)=\frac{I_{j}(a x)}{x I_{i}(b x)}\left(a x \frac{I_{j}^{\prime}(a x)}{I_{j}(x)}-b x \frac{I_{i}^{\prime}(a x)}{I_{i}(x)}\right)= \\
\frac{I_{j}(a x)}{x I_{i}(b x)}\left(\frac{u_{j}^{\prime}\left(z_{1}\right)}{z_{1} u_{j}^{\prime \prime}\left(z_{1}\right)}-\frac{u_{i}^{\prime}\left(z_{2}\right)}{z_{2} u_{i}^{\prime \prime}\left(z_{2}\right)}\right) \\
=\frac{z_{1}}{z_{2}}\left(\gamma_{i}\left(z_{2}\right)^{-1}-\gamma_{j}\left(z_{1}\right)^{-1}\right)>0 .
\end{array}
$$

Proof of Proposition 4.1: Suppose that $\gamma_{i}(x) \geq \gamma_{j}(y) \geq 1$, for all $x, y$. Then, by Lemma B.1, the function

$$
f(x)=\frac{I_{i}\left(\lambda_{i} x\right)}{I_{j}\left(\lambda_{j} x\right)}
$$

is increasing and $x I_{i}\left(\lambda_{i} x\right)$ is also increasing. Therefore,

$$
E\left[M I_{j}\left(\lambda_{j} M\right)\right] E[f(M)] \leq E\left[M I_{j}\left(\lambda_{j} M\right) f(M)\right]=E\left[M I_{i}\left(\lambda_{i} M\right)\right]
$$

that is

$$
\operatorname{Cov}\left(\frac{W_{i T}}{W_{j T}}, M W_{j T}\right) \geq 0
$$

and Lemma 4.1 implies

$$
E\left[\frac{W_{i T}}{W_{j T}}\right] \frac{W_{i 0}}{W_{k 0}} \leq 1
$$

Similarly, if $\gamma_{i}(x) \leq \gamma_{j}(y) \leq 1$ then both $f(x)$ and $x I_{i}\left(\lambda_{i} x\right)$ are decreasing and the same argument applies.

If $\gamma_{i}(x) \geq 1 \geq \gamma_{j}(x)$ then $x I_{j}\left(\lambda_{j} x\right)$ decreases and $f(x)$ increases, so that

$$
E\left[M I_{j}\left(\lambda_{j} M\right)\right] E[f(M)] \geq E\left[M I_{j}\left(\lambda_{j} M\right) f(M)\right]=E\left[M I_{i}\left(\lambda_{i} M\right)\right]
$$

and the claim (iii) follows. If $\gamma_{i}(x) \leq 1 \leq \gamma_{j}(x)$ then $x I_{j}\left(\lambda_{j} x\right)$ increases and $f(x)$ decreases, and the same argument applies.

Proof of Lemma 4.3: Let

$$
z_{i}=\psi_{i} E[D M] / E\left[M^{1-b_{i}}\right] .
$$

Then, the equilibrium equation is

$$
\sum_{i} z_{i} M^{-b_{i}}=D
$$


Suppose that

$$
M>\left(\sum_{i} D^{-\gamma_{i} / \Gamma} z_{i}^{\gamma_{i} / \Gamma}\right)^{\Gamma}
$$

Then,

$$
\sum_{i} z_{i} M^{-b_{i}} D^{-1}<\sum_{i} z_{i}\left(\sum_{i} D^{-\gamma_{i} / \Gamma} z_{i}^{\gamma_{i} / \Gamma}\right)^{-\Gamma / \gamma_{i}}=\sum_{i}\left(\frac{D^{-\gamma_{i} / \Gamma} z_{i}^{\gamma_{i} / \Gamma}}{\sum_{i} D^{-\gamma_{i} / \Gamma} z_{i}^{\gamma_{i} / \Gamma}}\right)^{\Gamma / \gamma_{i}}
$$

Since $\Gamma>\gamma_{i}$ for all $i$, we get

$$
\left(\frac{D^{-\gamma_{i} / \Gamma} z_{i}^{\gamma_{i} / \Gamma}}{\sum_{i} D^{-\gamma_{i} / \Gamma} z_{i}^{\gamma_{i} / \Gamma}}\right)^{\Gamma / \gamma_{i}}<\frac{D^{-\gamma_{i} / \Gamma} z_{i}^{\gamma_{i} / \Gamma}}{\sum_{i} D^{-\gamma_{i} / \Gamma} z_{i}^{\gamma_{i} / \Gamma}}
$$

and therefore

$$
\sum_{i} z_{i} M^{-b_{i}} D^{-1}<\sum_{i} \frac{D^{-\gamma_{i} / \Gamma} z_{i}^{\gamma_{i} / \Gamma}}{\sum_{i} D^{-\gamma_{i} / \Gamma} z_{i}^{\gamma_{i} / \Gamma}}=1
$$

which is a contradiction. The estimate from below follows by the same argument.

Lemma B.2 There exist constants $K_{1}, K_{2}>0$ such that

$$
K_{1} \sum_{i} \frac{E[D M]}{E\left[D^{1-\gamma_{i}}\right]} D^{-\gamma_{i}} \leq M \leq K_{2} \sum_{i} \frac{E[D M]}{E\left[D^{1-\gamma_{i}}\right]} D^{-\gamma_{i}}
$$

Proof: By Lemma 4.3,

$$
\frac{1}{n} \sum_{i} D^{-\gamma_{i}}\left(\psi_{i} E[D M] / E\left[M^{1-b_{i}}\right]\right)^{\gamma_{i}} \leq M \leq n \sum_{i} D^{-\gamma_{i}}\left(\psi_{i} E[D M] / E\left[M^{1-b_{i}}\right]\right)^{\gamma_{i}}
$$

By Lemma 4.4,

$$
K_{1} \frac{E[D M]}{E\left[D^{1-\gamma_{i}}\right]} \leq\left(\psi_{i} E[D M] / E\left[M^{1-b_{i}}\right]\right)^{\gamma_{i}} \leq K_{2} \frac{E[D M]}{E\left[D^{1-\gamma_{i}}\right]}
$$

for some $K_{1}, K_{2}>0$. The proof is complete.

We will also need

Lemma B.3 For any $\alpha>0$ and any $x_{i}>0$,

$$
\min \left\{n^{\alpha-1}, 1\right\} \sum_{i} x_{i}^{\alpha} \leq\left(\sum_{i} x_{i}\right)^{\alpha} \leq \max \left\{n^{\alpha-1}, 1\right\} \sum_{i} x_{i}^{\alpha} .
$$


Proof of Theorem 4.1: Suppose first that $\gamma_{i}>\gamma_{j}>1$. Then, Lemma 4.4 implies

$$
W_{i T}=\left(\lambda_{i} M\right)^{-b_{i}}=\frac{\psi_{i} E[D M]}{E\left[M^{1-b_{i}}\right]} M^{-b_{i}}
$$

and

$$
K_{1}\left(\frac{E[D M]}{E\left[D^{1-\gamma_{i}}\right]}\right)^{b_{i}} M^{-b_{i}} \leq W_{i T} \leq K_{2}\left(\frac{E[D M]}{E\left[D^{1-\gamma_{i}}\right]}\right)^{b_{i}} M^{-b_{i}} .
$$

Therefore, by Lemmas B.3 and Lemma B.2,

$$
\begin{gathered}
E\left[W_{i T} / W_{j T}\right] \leq K_{1} \frac{E\left[D^{1-\gamma_{j}}\right]^{b_{j}}}{E\left[D^{1-\gamma_{i}}\right]^{b_{i}}} E[D M]^{b_{i}-b_{j}} E\left[M^{b_{j}-b_{i}}\right] \\
\leq K_{2} \frac{E\left[D^{1-\gamma_{j}}\right]_{j}^{b_{j}}}{E\left[D^{1-\gamma_{i}}\right]^{b_{i}}} E[D M]^{b_{i}-b_{j}} E\left[\left(\sum_{k} \frac{E[D M]}{E\left[D^{1-\gamma_{k}}\right]} D^{-\gamma_{k}}\right)^{b_{j}-b_{i}}\right] \\
\leq K \sum_{k} \frac{E\left[D^{1-\gamma_{j}}\right]^{b_{j}} E\left[D^{\left(b_{i}-b_{j}\right) \gamma_{k}}\right]}{E\left[D^{1-\gamma_{i}}\right]^{b_{i}} E\left[D^{1-\gamma_{k}}\right]^{b_{j}-b_{i}}} \\
=K \sum_{k} e^{\frac{1}{2} \sigma^{2} T\left(\left(1-\gamma_{j}\right)^{2} b_{j}+\left(b_{i}-b_{j}\right)^{2} \gamma_{k}^{2}-\left(1-\gamma_{i}\right)^{2} b_{i}-\left(1-\gamma_{k}\right)^{2}\left(b_{j}-b_{i}\right)\right)} \\
e^{T \rho\left(\left(1-\gamma_{j}\right) b_{j}+\left(b_{i}-b_{j}\right) \gamma_{k}-\left(1-\gamma_{i}\right) b_{i}-\left(1-\gamma_{k}\right)\left(b_{j}-b_{i}\right)\right)} \\
=K \sum_{k} e^{\frac{1}{2} \sigma^{2} T\left(\left(1-\gamma_{j}\right)^{2} b_{j}+\left(b_{i}-b_{j}\right)^{2} \gamma_{k}^{2}-\left(1-\gamma_{i}\right)^{2} b_{i}-\left(1-\gamma_{k}\right)^{2}\left(b_{j}-b_{i}\right)\right) .}
\end{gathered}
$$

Thus, it suffices to show that

$$
\left(1-\gamma_{j}\right)^{2} b_{j}+\left(b_{i}-b_{j}\right)^{2} \gamma_{k}^{2}-\left(1-\gamma_{i}\right)^{2} b_{i}-\left(1-\gamma_{k}\right)^{2}\left(b_{j}-b_{i}\right)<0
$$

for all $k$. We have

$$
\begin{gathered}
\left(1-\gamma_{j}\right)^{2} b_{j}+\left(b_{i}-b_{j}\right)^{2} \gamma_{k}^{2}-\left(1-\gamma_{i}\right)^{2} b_{i}-\left(1-\gamma_{k}\right)^{2}\left(b_{j}-b_{i}\right) \\
=\left(b_{j}-1\right)^{2} b_{j}^{-1}-\left(b_{i}-1\right)^{2} b_{i}^{-1}+\left(b_{j}-b_{i}\right)\left(\left(b_{j}-b_{i}\right) \gamma_{k}^{2}-\left(1-\gamma_{k}\right)^{2}\right) \\
=\left(b_{j}-b_{i}\right)\left(1-\left(b_{i} b_{j}\right)^{-1}+\left(b_{j}-b_{i}\right) \gamma_{k}^{2}-\left(1-\gamma_{k}\right)^{2}\right) \\
=\left(b_{j}-b_{i}\right) b_{j} b_{i}\left(\gamma_{i} \gamma_{j}-\gamma_{i}^{2} \gamma_{j}^{2}+\left(\gamma_{i}-\gamma_{j}\right) \gamma_{k}^{2}-\gamma_{i} \gamma_{j}\left(1-\gamma_{k}\right)^{2}\right) .
\end{gathered}
$$

The function

$$
f\left(\gamma_{k}\right)=\gamma_{i} \gamma_{j}-\gamma_{i}^{2} \gamma_{j}^{2}+\left(\gamma_{i}-\gamma_{j}\right) \gamma_{k}^{2}-\gamma_{i} \gamma_{j}\left(1-\gamma_{k}\right)^{2}
$$

attains its maximum at

$$
\gamma_{k}=\frac{\gamma_{i} \gamma_{j}}{\gamma_{i} \gamma_{j}+\gamma_{j}-\gamma_{i}}
$$


Thus, we need to show that

$$
\begin{aligned}
0>\gamma_{i} \gamma_{j}-\gamma_{i}^{2} \gamma_{j}^{2}+\left(\gamma_{i}-\gamma_{j}\right)\left(\frac{\gamma_{i} \gamma_{j}}{\gamma_{i} \gamma_{j}+\gamma_{j}-\gamma_{i}}\right)^{2}-\gamma_{i} \gamma_{j}\left(1-\frac{\gamma_{i} \gamma_{j}}{\gamma_{i} \gamma_{j}+\gamma_{j}-\gamma_{i}}\right)^{2} \\
=\gamma_{i} \gamma_{j}-\gamma_{i}^{2} \gamma_{j}^{2}+\left(\gamma_{i}-\gamma_{j}\right)\left(\frac{\gamma_{i} \gamma_{j}}{\gamma_{i} \gamma_{j}+\gamma_{j}-\gamma_{i}}\right)^{2}-\gamma_{i} \gamma_{j}\left(1-\frac{\gamma_{i} \gamma_{j}}{\gamma_{i} \gamma_{j}+\gamma_{j}-\gamma_{i}}\right)^{2} \\
=\frac{\gamma_{i} \gamma_{j}}{\left(\gamma_{i} \gamma_{j}+\gamma_{j}-\gamma_{i}\right)^{2}}\left(\left(1-\gamma_{i} \gamma_{j}\right)\left(\gamma_{i} \gamma_{j}+\gamma_{j}-\gamma_{i}\right)^{2}+\left(\gamma_{i}-\gamma_{j}\right) \gamma_{i} \gamma_{j}-\left(\gamma_{i}-\gamma_{j}\right)^{2}\right) .
\end{aligned}
$$

We have

$$
\begin{aligned}
&\left(1-\gamma_{i} \gamma_{j}\right)\left(\gamma_{i} \gamma_{j}+\gamma_{j}-\gamma_{i}\right)^{2}+\left(\gamma_{i}-\gamma_{j}\right) \gamma_{i} \gamma_{j}-\left(\gamma_{i}-\gamma_{j}\right)^{2} \\
&=\left(1-\gamma_{i} \gamma_{j}\right)\left(\gamma_{i} \gamma_{j}+\gamma_{j}-\gamma_{i}\right)^{2}+\left(\gamma_{i}-\gamma_{j}\right)\left(\gamma_{i} \gamma_{j}-\gamma_{i}+\gamma_{j}\right) \\
& \quad=\left(\gamma_{i}\left(\gamma_{j}-1\right)+\gamma_{j}\right)\left(\left(1-\gamma_{i} \gamma_{j}\right)\left(\gamma_{i} \gamma_{j}+\gamma_{j}-\gamma_{i}\right)+\gamma_{i}-\gamma_{j}\right)
\end{aligned}
$$

Hence, we need to show that

$$
0>\gamma_{i} \gamma_{j}+\gamma_{j}-\gamma_{i}-\gamma_{i}^{2} \gamma_{j}^{2}-\gamma_{i} \gamma_{j}^{2}+\gamma_{i}^{2} \gamma_{j}+\gamma_{i}-\gamma_{j}=\gamma_{i} \gamma_{j}\left(1-\gamma_{j}\right)\left(1+\gamma_{i}\right)
$$

which follows from $\gamma_{j}>1$.

Let now $\gamma_{i}<\gamma_{j}<1$. Then, by Lemma B.2,

$$
M \geq K_{1}\left(\frac{E[D M]}{E\left[D^{1-\gamma_{i}}\right]} D^{-\gamma_{i}}+\frac{E[D M]}{E\left[D^{1-\gamma_{i}}\right]} D^{-\gamma_{j}}\right)
$$

and therefore

$$
\begin{aligned}
& E\left[W_{i T} / W_{j T}\right] \leq K_{1} \frac{E\left[D^{1-\gamma_{j}}\right]^{b_{j}}}{E\left[D^{1-\gamma_{i}}\right]^{b_{i}}} E[D M]^{b_{i}-b_{j}} E\left[M^{b_{j}-b_{i}}\right] \\
& \leq K \frac{E\left[D^{1-\gamma_{j}}\right]^{b_{j}}}{E\left[D^{1-\gamma_{i}}\right]^{b_{i}}} E[D M]^{b_{i}-b_{j}} E\left[\left(\frac{E[D M]}{E\left[D^{1-\gamma_{i}}\right]} D^{-\gamma_{i}}+\frac{E[D M]}{E\left[D^{1-\gamma_{i}}\right]} D^{-\gamma_{j}}\right)^{b_{j}-b_{i}}\right] \\
& \quad=K \frac{E\left[D^{1-\gamma_{j}}\right]^{b_{j}}}{E\left[D^{1-\gamma_{i}}\right]^{b_{i}}} E\left[\left(\frac{D^{-\gamma_{i}}}{E\left[D^{1-\gamma_{i}}\right]}+\frac{D^{-\gamma_{j}}}{E\left[D^{1-\gamma_{j}}\right]}\right)^{b_{j}-b_{i}}\right] .
\end{aligned}
$$

Consider now an artificial economy, populated only by funds $i$ and $j$. Then, the equilibrium SDF $\tilde{M}$ in this economy solves

$$
\frac{\psi_{i} E[\tilde{M} D]}{E\left[\tilde{M}^{1-b_{i}}\right]} \tilde{M}^{-b_{i}}+\frac{\psi_{j} E[\tilde{M} D]}{E\left[\tilde{M}^{1-b_{j}}\right]} \tilde{M}^{-b_{j}}=\left(\psi_{i}+\psi_{j}\right) D
$$

and, by Lemma B.2, there exist constants $K_{1}, K_{2}$ such that

$$
K_{1}\left(\frac{E[D \tilde{M}]}{E\left[D^{1-\gamma_{i}}\right]} D^{-\gamma_{i}}+\frac{E[D \tilde{M}]}{E\left[D^{1-\gamma_{j}}\right]} D^{-\gamma_{j}}\right) \leq \tilde{M} \leq K_{2}\left(\frac{E[D \tilde{M}]}{E\left[D^{1-\gamma_{i}}\right]} D^{-\gamma_{i}}+\frac{E[D \tilde{M}]}{E\left[D^{1-\gamma_{j}}\right]} D^{-\gamma_{j}}\right)
$$


and both

$$
\frac{E[D \tilde{M}]^{1-\gamma_{i}} E\left[\tilde{M}^{1-b_{i}}\right]^{\gamma_{i}}}{E\left[D^{1-\gamma_{i}}\right]}, \frac{E[D \tilde{M}]^{1-\gamma_{j}} E\left[\tilde{M}^{1-b_{j}}\right]^{\gamma_{j}}}{E\left[D^{1-\gamma_{j}}\right]}
$$

belong to the interval $\left[K_{1}, K_{2}\right]$. Therefore,

$$
\begin{array}{r}
\frac{E\left[D^{1-\gamma_{j}}\right]^{b_{j}}}{E\left[D^{1-\gamma_{i}}\right]^{b_{i}}} E\left[\left(\frac{D^{-\gamma_{i}}}{E\left[D^{1-\gamma_{i}}\right]}+\frac{D^{-\gamma_{j}}}{E\left[D^{1-\gamma_{j}}\right]}\right)^{b_{j}-b_{i}}\right] \leq K \frac{E\left[\tilde{M}^{b_{j}-b_{i}}\right] E\left[\tilde{M}^{1-b_{j}}\right]}{E\left[\tilde{M}^{1-b_{i}}\right]} \\
\leq \tilde{K} \frac{E\left[\hat{M}^{b_{j}-b_{i}}\right] E\left[\hat{M}^{1-b_{j}}\right]}{E\left[\hat{M}^{1-b_{i}}\right]}
\end{array}
$$

with

$$
\hat{M}=\frac{1}{E\left[D^{1-\gamma_{i}}\right]} D^{-\gamma_{i}}+\frac{1}{E\left[D^{1-\gamma_{j}}\right]} D^{-\gamma_{j}}
$$

Introduce a new measure

$$
d Q=\frac{D^{1-\gamma_{i}} d P}{E\left[D^{1-\gamma_{i}}\right]}
$$

Note now that

$$
\hat{M} D^{\gamma_{i}}=f_{T}(D)
$$

is a monotone decreasing function of $D$ and, under this new measure,

$$
\begin{aligned}
& \frac{E\left[\hat{M}^{b_{j}-b_{i}}\right] E\left[\hat{M}^{1-b_{j}}\right]}{E\left[\hat{M}^{1-b_{i}}\right]} \\
& =E\left[D^{1-\gamma_{i}}\right] \frac{E^{Q}\left[f_{T}(D)^{b_{j}-b_{i}} D^{-\gamma_{i}\left(b_{j}-b_{i}\right)-1+\gamma_{i}}\right] E^{Q}\left[f_{T}(D)^{1-b_{j}} D^{-\gamma_{i}\left(1-b_{j}\right)-1+\gamma_{i}}\right]}{E^{Q}\left[f_{T}(D)^{1-b_{i}} D^{-\gamma_{i}\left(1-b_{i}\right)-1+\gamma_{i}}\right]} \\
& =E\left[D^{1-\gamma_{i}}\right] \frac{E^{Q}\left[f_{T}(D)^{b_{j}-b_{i}} D^{-\gamma_{i} b_{j}+\gamma_{i}}\right] E^{Q}\left[f_{T}(D)^{1-b_{j}} D^{\gamma_{i} b_{j}-1}\right]}{E^{Q}\left[f_{T}(D)^{1-b_{i}}\right]} .
\end{aligned}
$$

Since $\gamma_{i}<\gamma_{j}<1, b_{i}>b_{j}>1$ and therefore $f_{T}(D)^{b_{j}-b_{i}}$ is an increasing function of $D$, whereas $D^{-\gamma_{i} b_{j}+\gamma_{i}}$ is decreasing in $D$. Therefore, Lemma 4.2 implies that

$$
E^{Q}\left[f_{T}(D)^{b_{j}-b_{i}} D^{-\gamma_{i} b_{j}+\gamma_{i}}\right] \leq E^{Q}\left[f_{T}(D)^{b_{j}-b_{i}}\right] E^{Q}\left[D^{-\gamma_{i} b_{j}+\gamma_{i}}\right] .
$$

Similarly, $f_{T}(D)^{1-b_{j}}$ is increasing in $D$, whereas $\gamma_{i} b_{j}-1<\gamma_{j} b_{j}-1=0$, that is $D^{\gamma_{i} b_{j}-1}$ is decreasing in $D$ and Lemma 4.2 implies

$$
E^{Q}\left[f_{T}(D)^{1-b_{j}} D^{\gamma_{i} b_{j}-1}\right] \leq E^{Q}\left[f_{T}(D)^{1-b_{j}}\right] E^{Q}\left[D^{\gamma_{i} b_{j}-1}\right]
$$

Similarly, Lemma 4.2 implies

$$
E^{Q}\left[f_{T}(D)^{b_{j}-b_{i}}\right] E^{Q}\left[f_{T}(D)^{1-b_{j}}\right] \leq E^{Q}\left[f_{T}(D)^{1-b_{i}}\right]
$$


Combining (46)-(48), we get

$$
\begin{gathered}
E\left[D^{1-\gamma_{i}}\right] \frac{E^{Q}\left[f_{T}(D)^{b_{j}-b_{i}} D^{-\gamma_{i} b_{j}+\gamma_{i}}\right] E^{Q}\left[f_{T}(D)^{1-b_{j}} D^{\gamma_{i} b_{j}-1}\right]}{E^{Q}\left[f_{T}(D)^{1-b_{i}}\right]} \\
\leq E\left[D^{1-\gamma_{i}}\right] \frac{E^{Q}\left[f_{T}(D)^{b_{j}-b_{i}}\right] E^{Q}\left[D^{-\gamma_{i} b_{j}+\gamma_{i}}\right] E^{Q}\left[f_{T}(D)^{1-b_{j}}\right] E^{Q}\left[D^{\gamma_{i} b_{j}-1}\right]}{E^{Q}\left[f_{T}(D)^{1-b_{i}}\right]} \\
\leq E\left[D^{1-\gamma_{i}}\right] E^{Q}\left[D^{-\gamma_{i} b_{j}+\gamma_{i}}\right] E^{Q}\left[D^{\gamma_{i} b_{j}-1}\right]=\frac{E\left[D^{1-\gamma_{i} b_{j}}\right] E\left[D^{\gamma_{i} b_{j}-\gamma_{i}}\right]}{E\left[D^{1-\gamma_{i}}\right]} \\
=e^{\frac{1}{2} \sigma^{2} T\left(\left(1-\gamma_{i} b_{j}\right)^{2}+\left(\gamma_{i} b_{j}-\gamma_{i}\right)^{2}-\left(1-\gamma_{i}\right)^{2}\right)+\rho T\left(1-\gamma_{i} b_{j}+\gamma_{i} b_{j}-\gamma_{i}-\left(1-\gamma_{i}\right)\right)}=e^{-\frac{1}{2} \sigma^{2} T\left(1-\gamma_{i} b_{j}\right)\left(\gamma_{i} b_{j}-\gamma_{i}\right)}
\end{gathered}
$$

and (43)-(45) imply the required convergence.

Proof of Proposition 4.2: Let $\gamma_{i}<\gamma_{j}$. By Lemma B.2,

$$
M \geq K_{1} \frac{E[D M]}{E\left[D^{1-\gamma_{j}}\right]} D^{-\gamma_{j}}
$$

Combining this bound with (37), we get

$$
\begin{aligned}
W_{i T} / W_{j T} \leq K_{1} \frac{E\left[D^{1-\gamma_{j}}\right]^{b_{j}}}{E\left[D^{1-\gamma_{i}}\right]^{b_{i}}} E[D M]^{b_{i}-b_{j}} M^{b_{j}-b_{i}} & \leq K \frac{E\left[D^{1-\gamma_{j}}\right]^{b_{j}}}{E\left[D^{1-\gamma_{i}}\right]^{b_{i}}}\left(\frac{D^{-\gamma_{j}}}{E\left[D^{1-\gamma_{j}}\right]}\right)^{b_{j}-b_{i}} \\
& =K e^{\frac{1}{2} \sigma^{2} T\left(\left(1-\gamma_{j}\right)^{2}-\left(1-\gamma_{i}\right)^{2}\right) b_{i}+\sigma \gamma_{j}\left(b_{i}-b_{j}\right) B_{T}} .
\end{aligned}
$$

The right-hand side converges to zero almost surely by the law of large numbers as soon as $\left|1-\gamma_{j}\right|<\left|1-\gamma_{i}\right|$.

Let now $\gamma_{i}>\gamma_{j}$. By $(37)$,

$$
\hat{K}_{2} \leq \frac{W_{i T} / W_{j T}}{\frac{E\left[D^{1-\gamma_{j}}\right]^{b_{j}}}{E\left[D^{1-\gamma_{i}}\right]^{b_{i}}} E[D M]^{b_{i}-b_{j}} M^{b_{j}-b_{i}}} \leq \hat{K}_{1} .
$$

Combining Lemma B.2 and B.3, we get that

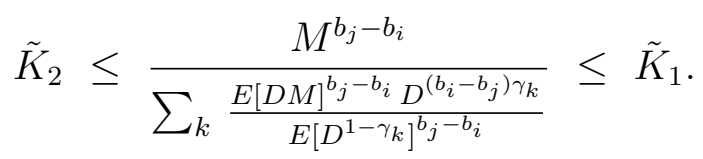

and therefore $W_{i T} / W_{j T}$ converges to zero (infinity) if and only if so does

$$
\begin{aligned}
K_{1} \sum_{k} \frac{E\left[D^{1-\gamma_{j}}\right]^{b_{j}} D^{\left(b_{i}-b_{j}\right) \gamma_{k}}}{E\left[D^{1-\gamma_{i}}\right]^{b_{i}} E\left[D^{1-\gamma_{k}}\right]^{b_{j}-b_{i}}} & \\
& =K_{1} \sum_{k} e^{\frac{1}{2} \sigma^{2} T\left(\left(1-\gamma_{j}\right)^{2} b_{j}-\left(1-\gamma_{i}\right)^{2} b_{i}-\left(1-\gamma_{k}\right)^{2}\left(b_{j}-b_{i}\right)+\sigma \gamma_{k}\left(b_{i}-b_{j}\right) B_{T}\right) .}
\end{aligned}
$$

The claim follows from the LLN. It remains to show that (6) always holds if $\gamma_{i}>\gamma_{j}>1$. This follows from (39). 


\section{Proofs for Section 5}

In order to prove Theorem 5.1, we need a sequence of technical lemmas.

Lemma C.1 The function

$$
f_{a}\left(x_{1}, \cdots, x_{n}\right)=\left(\sum_{i} x_{i}^{1 / a}\right)^{a}
$$

is jointly concave in $\left(x_{1}, \cdots, x_{n}\right)$ if $a \geq 1$ and jointly convex if $a<1$. Furthermore, $f_{a}$ is monotone increasing in a.

Proof: Let $a>1$. The Hessian $H(f)$ of the function $f$ is given by

$$
\begin{aligned}
H(f) & =\left(\partial^{2} f / \partial x_{i} \partial x_{j}\right)_{i, j=1}^{n} \\
& =\left(1-a^{-1}\right)\left(\sum_{i=1}^{n} x_{i}^{1 / a}\right)^{a-2}\left(\left(x_{i}^{1 / a-1} x_{j}^{1 / a-1}\right)_{i, j=1}^{n}\right. \\
& \left.-\left(\sum_{i=1}^{n} x_{i}^{1 / a}\right) \operatorname{diag}\left(x_{i}^{1 / a-2}\right)_{i=1}^{n}\right) \\
& =\left(1-a^{-1}\right) f^{1-2 / a} \operatorname{diag}\left(x_{i}^{a^{-1} / 2-1}\right)_{i=1}^{n}\left(\left(x_{i}^{a^{-1} / 2} x_{j}^{a^{-1} / 2}\right)_{i, j=1}^{n}\right. \\
& \left.-f^{1 / a} I\right) \operatorname{diag}\left(x_{i}^{a^{-1} / 2-1}\right)_{i=1}^{n} .
\end{aligned}
$$

Here, $I$ is the identity matrix. The matrix

$$
A=\left(x_{i}^{a^{-1} / 2} x_{j}^{a^{-1} / 2}\right)_{i, j=1}^{n}
$$

equals $f^{1 / a}$ times the orthogonal projection onto the vector $\left(x_{i}^{a^{-1} / 2}\right)_{i=1}^{n}$. Thus, $\|A\|=f^{1 / a}$ and the matrix

$$
\left(x_{i}^{a^{-1} / 2} x_{j}^{a^{-1} / 2}\right)_{i, j=1}^{n}-f^{1 / a} I
$$

is negative definite. Therefore, $H(f)$ is also negative definite. The case $a<1$ is similar.

It remains to prove monotonicity. Let $a<b$. Then,

$$
\frac{\left(\sum_{i} x_{i}^{1 / a}\right)^{a}}{\left(\sum_{i} x_{i}^{1 / b}\right)^{b}}=\left(\sum_{i}\left(\frac{x_{i}}{\left(\sum_{i} x_{i}^{1 / b}\right)^{b}}\right)^{1 / a}\right)^{a}=\left(\sum_{i}\left(\frac{x_{i}^{1 / b}}{\left(\sum_{i} x_{i}^{1 / b}\right)}\right)^{b / a}\right)^{a}
$$

Since

$$
\frac{x_{i}^{1 / b}}{\left(\sum_{i} x_{i}^{1 / b}\right)}<1
$$


for all $i$ and $b / a>1$, we get

$$
\left(\frac{x_{i}^{1 / b}}{\left(\sum_{i} x_{i}^{1 / b}\right)}\right)^{b / a}<\frac{x_{i}^{1 / b}}{\left(\sum_{i} x_{i}^{1 / b}\right)}
$$

and hence

$$
\left(\sum_{i}\left(\frac{x_{i}^{1 / b}}{\left(\sum_{i} x_{i}^{1 / b}\right)}\right)^{b / a}\right)^{a}<\left(\sum_{i} \frac{x_{i}^{1 / b}}{\left(\sum_{i} x_{i}^{1 / b}\right)}\right)^{a}=1 .
$$

Lemma C.2 For any $\alpha \in \mathbb{R}$, we have

$$
\begin{aligned}
\left(\sum _ { i = 1 } ^ { n } E _ { t } [ D ^ { \alpha - \gamma _ { i } } ] ^ { 1 / \gamma } \left(\psi_{i} E[D M] /\right.\right. & \left.\left.E\left[M^{1-b_{i}}\right]\right)^{\gamma_{i} / \gamma}\right)^{\gamma} \leq E_{t}\left[D^{\alpha} M\right] \\
& \leq\left(\sum_{i} E_{t}\left[D^{\alpha-\gamma_{i}}\right]^{1 / \Gamma}\left(\psi_{i} E[D M] / E\left[M^{1-b_{i}}\right]\right)^{\gamma_{i} / \Gamma}\right)^{\Gamma}
\end{aligned}
$$

Proof: Using Lemma 4.3, we get

$$
\begin{aligned}
E_{t}\left[\left(\sum_{i} D^{\left(\alpha-\gamma_{i}\right) / \gamma}\left(\psi_{i} E[D M] / E\left[M^{1-b_{i}}\right]\right)^{\gamma_{i} / \gamma}\right)^{\gamma}\right] \leq E_{t}\left[D^{\alpha} M\right] \\
\leq E_{t}\left[\left(\sum_{i} D^{\left(\alpha-\gamma_{i}\right) / \Gamma}\left(\psi_{i} E[D M] / E\left[M^{1-b_{i}}\right]\right)^{\gamma_{i} / \Gamma}\right)^{\Gamma}\right] .
\end{aligned}
$$

By Lemma C.1,

$$
\begin{gathered}
\left(\sum_{i}\left(E_{t}\left[X_{i}\right]\right)^{1 / \gamma}\right)^{\gamma} \leq E_{t}\left[\left(\sum_{i} X_{i}^{1 / \gamma}\right)^{\gamma}\right] \\
\leq E_{t}\left[\left(\sum_{i} X_{i}^{1 / \Gamma}\right)^{\Gamma}\right] \leq\left(\sum_{i}\left(E_{t}\left[X_{i}\right]\right)^{1 / \Gamma}\right)^{\Gamma}
\end{gathered}
$$

for any positive random variables $X_{i}$. Applying (54) to the random variables

$$
X_{i}=D^{\alpha-\gamma_{i}}\left(\psi_{i} E[D M] / E\left[M^{1-b_{i}}\right]\right)^{\gamma_{i}}
$$

we get the stated result. 
Lemma C.3 Let $\gamma_{0}$ be the risk aversion closest to one. Let $t=\lambda T$. For any

$$
\lambda \in \Pi_{i}
$$

we have

$$
\lim _{T \rightarrow \infty} \frac{E_{t}[D M]}{\left(\psi_{0} E[D M] E\left[M^{1-b_{0}}\right]^{-1}\right)^{\gamma_{0}} E_{t}\left[D^{1-\gamma_{0}}\right]}=1 .
$$

Proof: By (52),

$$
\begin{aligned}
\left(\sum_{i} E_{t}\left[D^{1-\gamma_{i}}\right]^{1 / \gamma}\left(\psi_{i} E[D M] / E\left[M^{1-b_{i}}\right]\right)^{\gamma_{i} / \gamma}\right)^{\gamma} \leq E_{t}[D M] & \\
& \leq\left(\sum_{i} E_{t}\left[D^{1-\gamma_{i}}\right]^{1 / \Gamma}\left(\psi_{i} E[D M] / E\left[M^{1-b_{i}}\right]\right)^{\gamma_{i} / \Gamma}\right)^{\Gamma} .
\end{aligned}
$$

Dividing (55)by

$$
E_{t}\left[D^{1-\gamma_{0}}\right]\left(\psi_{0} E[D M] E\left[M^{1-b_{0}}\right]^{-1}\right)^{\gamma_{0}}
$$

and observing that

$$
\frac{E_{t}\left[D^{1-\gamma_{i}}\right]\left(\psi_{i} E[D M] / E\left[M^{1-b_{i}}\right]\right)^{\gamma_{i}}}{E_{t}\left[D^{1-\gamma_{0}}\right]\left(\psi_{0} E[D M] / E\left[M^{1-b_{0}}\right]^{-1}\right)^{\gamma_{0}}}=\frac{E_{t}\left[D^{1-\gamma_{i}}\right]}{E_{t}\left[D^{1-\gamma_{0}}\right]} \frac{E[D M]^{1-\gamma_{0}} E\left[M^{1-b_{0}}\right]^{\gamma_{0}}}{E[D M]^{1-\gamma_{i}} E\left[M^{1-b_{i}}\right]^{\gamma_{i}}}
$$

we arrive at

$$
\begin{aligned}
\left(1+\sum_{i \neq 0}\left(\frac{\psi_{i}^{\gamma_{i}}}{\psi_{0}^{\gamma_{0}}} \frac{E_{t}\left[D^{1-\gamma_{i}}\right]}{E_{t}\left[D^{1-\gamma_{0}}\right]} \frac{E[D M]^{1-\gamma_{0}} E\left[M^{1-b_{0}}\right]^{\gamma_{0}}}{E[D M]^{1-\gamma_{i}} E\left[M^{1-b_{i}}\right]^{\gamma_{i}}}\right)^{1 / \gamma}\right)^{\gamma} & \\
\leq & \frac{E_{t}[D M]}{\left(\psi_{0} E[D M] E\left[M^{1-b_{0}}\right]^{-1}\right)^{\gamma_{0}} E_{t}\left[D^{1-\gamma_{0}}\right]} \\
\leq & \left(1+\sum_{i \neq 0}\left(\frac{\psi_{i}^{\gamma_{i}}}{\psi_{0}^{\gamma_{0}}} \frac{E_{t}\left[D^{1-\gamma_{i}}\right]}{E_{t}\left[D^{1-\gamma_{0}}\right]} \frac{E[D M]^{1-\gamma_{0}} E\left[M^{1-b_{0}}\right]^{\gamma_{0}}}{E[D M]^{1-\gamma_{i}} E\left[M^{1-b_{i}}\right]^{\gamma_{i}}}\right)^{1 / \Gamma}\right)^{\Gamma} .
\end{aligned}
$$

By Lemma 4.4,

$$
K_{1} \frac{E\left[D^{1-\gamma_{0}}\right]}{E\left[D^{1-\gamma_{i}}\right]} \leq \frac{E[D M]^{1-\gamma_{0}} E\left[M^{1-b_{0}}\right]^{\gamma_{0}}}{E[D M]^{1-\gamma_{i}} E\left[M^{1-b_{i}}\right]^{\gamma_{i}}} \leq K_{2} \frac{E\left[D^{1-\gamma_{0}}\right]}{E\left[D^{1-\gamma_{i}}\right]}
$$

for some $K_{1}, K_{2}>0$ and therefore

$$
\begin{gathered}
\left(1+K_{1} \sum_{i \neq 0}\left(\frac{E_{t}\left[D^{1-\gamma_{i}}\right] E\left[D^{1-\gamma_{0}}\right]}{E_{t}\left[D^{1-\gamma_{0}}\right] E\left[D^{1-\gamma_{i}}\right]}\right)^{1 / \gamma}\right)^{\gamma} \\
\leq \frac{E_{t}[D M]}{\left(\psi_{0} E[D M] E\left[M^{1-b_{0}}\right]^{-1}\right)^{\gamma_{0}} E_{t}\left[D^{1-\gamma_{0}}\right]} \\
\leq\left(1+K_{2} \sum_{i \neq 0}\left(\frac{E_{t}\left[D^{1-\gamma_{i}}\right] E\left[D^{1-\gamma_{0}}\right]}{E_{t}\left[D^{1-\gamma_{0}}\right] E\left[D^{1-\gamma_{i}}\right]}\right)^{1 / \Gamma}\right)^{\Gamma} .
\end{gathered}
$$


Furthermore,

$$
\frac{E_{t}\left[D^{1-\gamma_{i}}\right] E\left[D^{1-\gamma_{0}}\right]}{E_{t}\left[D^{1-\gamma_{0}}\right] E\left[D^{1-\gamma_{i}}\right]}=e^{\sigma\left(\gamma_{0}-\gamma_{i}\right) B_{t}} e^{\frac{1}{2} \sigma^{2}\left(\left|1-\gamma_{0}\right|^{2}-\left|1-\gamma_{i}\right|^{2}\right) t}
$$

converges to zero almost surely and the proof is complete.

Lemma C.4 Let $t=\lambda T$. For any

$$
\lambda \in \Pi_{i}
$$

we have

$$
\lim _{T \rightarrow \infty} \frac{E_{t}[M]}{\left(\psi_{i} E[D M] E\left[M^{1-b_{i}}\right]^{-1}\right)^{\gamma_{i}} E_{t}\left[D^{-\gamma_{i}}\right]}=1
$$

Proof: By (52),

$$
\begin{aligned}
\left(\sum_{j} E_{t}\left[D^{-\gamma_{j}}\right]^{1 / \gamma}\left(\psi_{j} E[D M] / E\left[M^{1-b_{j}}\right]\right)^{\gamma_{j} / \gamma}\right)^{\gamma} \leq E_{t}[M] & \\
& \leq\left(\sum_{j} E_{t}\left[D^{-\gamma_{j}}\right]^{1 / \Gamma}\left(\psi_{j} E[D M] / E\left[M^{1-b_{j}}\right]\right)^{\gamma_{j} / \Gamma}\right)^{\Gamma} .
\end{aligned}
$$

In complete analogy with (57), we get

$$
\begin{aligned}
&\left(1+K_{1} \sum_{j \neq i}\left(\frac{E_{t}\left[D^{-\gamma_{j}}\right] E\left[D^{1-\gamma_{i}}\right]}{E_{t}\left[D^{-\gamma_{i}}\right] E\left[D^{1-\gamma_{j}}\right]}\right)^{1 / \gamma}\right)^{\gamma} \\
& \leq \frac{E_{t}[D M]}{\left(\psi_{i} E[D M] E\left[M^{1-b_{i}}\right]^{-1}\right)^{\gamma_{i}} E_{t}\left[D^{-\gamma_{i}}\right]} \\
& \leq\left(1+K_{2} \sum_{j \neq i}\left(\frac{E_{t}\left[D^{-\gamma_{j}}\right] E\left[D^{1-\gamma_{i}}\right]}{E_{t}\left[D^{-\gamma_{i}}\right] E\left[D^{1-\gamma_{j}}\right]}\right)^{1 / \Gamma}\right)^{\Gamma}
\end{aligned}
$$

However, we have

$$
\frac{E_{t}\left[D^{-\gamma_{j}}\right] E\left[D^{1-\gamma_{i}}\right]}{E_{t}\left[D^{-\gamma_{i}}\right] E\left[D^{1-\gamma_{j}}\right]}=e^{\sigma\left(\gamma_{i}-\gamma_{j}\right) B_{t}} e^{\frac{1}{2} \sigma^{2} T\left(\left(\gamma_{j}^{2}-\gamma_{i}^{2}\right)(1-\lambda)+\left(1-\gamma_{i}\right)^{2}-\left(1-\gamma_{j}\right)^{2}\right)}
$$

and the claim follows if

$$
\gamma_{j}^{2}(1-\lambda)-\left(1-\gamma_{j}\right)^{2}<\gamma_{i}^{2}(1-\lambda)-\left(1-\gamma_{i}\right)^{2}
$$

for all $j \neq i$. Now, the function

$$
h(\gamma)=\gamma^{2}(1-\lambda)-(1-\gamma)^{2}=-\lambda \gamma^{2}+2 \gamma-1
$$

is a concave parabola, attaining maximum at the vertex $1 / \lambda$. Therefore, (60) holds if $\gamma_{i}$ is the closest to $1 / \lambda$ among all risk aversions. Let $0<i<l$. Then,

$$
\frac{\gamma_{i-1}+\gamma_{i}}{2}<\frac{1}{\lambda}<\frac{\gamma_{i}+\gamma_{i+1}}{2}
$$


and therefore, since $\gamma_{i-1}<\gamma_{i}<\gamma_{i+1}$, the claim is immediate. The cases $i=0, l$ are analogous.

Proof of Theorem 5.1: Let

$$
f_{i}(T)=\frac{\psi_{0}^{\gamma_{0}} E[D M]^{1-\gamma_{i}} E\left[M^{1-b_{i}}\right]^{\gamma_{i}} E\left[D^{1-\gamma_{0}}\right]}{\psi_{i}^{\gamma_{i}} E[D M]^{1-\gamma_{0}} E\left[M^{1-b_{0}}\right]^{\gamma_{0}} E\left[D^{1-\gamma_{i}}\right]}
$$

By the discussion before Theorem 5.1, $\gamma_{i}>1$ and therefore Lemma 4.4 implies

$$
\psi_{i}^{\gamma_{i}-1} \leq \frac{E[D M]^{1-\gamma_{i}} E\left[M^{1-b_{i}}\right]^{\gamma_{i}}}{E\left[D^{1-\gamma_{i}}\right]} \leq 1
$$

and

$$
\min \left\{\psi_{0}^{1-\gamma_{0}}, 1\right\} \leq \frac{E\left[D^{1-\gamma_{0}}\right]}{E[D M]^{1-\gamma_{0}} E\left[M^{1-b_{0}}\right]^{\gamma_{0}}} \leq \max \left\{\psi_{0}^{1-\gamma_{0}}, 1\right\}
$$

Hence, $f_{i}(T)$ satisfies the required estimate and, by Lemmas C.3 and C.4,

$$
S_{t} \sim e^{r(t-T)} \frac{\left(\psi_{0} E[D M] E\left[M^{1-b_{0}}\right]^{-1}\right)^{\gamma_{0}} E_{t}\left[D^{1-\gamma_{0}}\right]}{\left(\psi_{i} E[D M] E\left[M^{1-b_{i}}\right]^{-1}\right)^{\gamma_{i}} E_{t}\left[D^{-\gamma_{i}}\right]}=f_{i}\left(t \lambda^{-1}\right) e^{r(t-T)} \frac{E_{t}\left[D^{1-\gamma_{0}}\right] E\left[D^{1-\gamma_{i}}\right]}{E_{t}\left[D^{-\gamma_{i}}\right] E\left[D^{1-\gamma_{0}}\right]}
$$

and the claim follows. The case $i=0$ is analogous.

It remains to show that

$$
\left(P_{i \lambda_{i} T} e^{-r\left(\lambda_{i}^{-1}-1\right) T}\right) /\left(P_{i+1 \lambda_{i+1} T} e^{-r\left(\lambda_{i+1}^{-1}-1\right) T}\right) . \rightarrow+\infty .
$$

That is, we need to show that, if $\lambda_{i} \in \Pi_{i}$ and $\lambda_{i+1} \in \Pi_{i+1}$ then

$$
\begin{aligned}
\left(\left(1-\gamma_{0}\right)^{2}-\gamma_{i}^{2}\right)\left(1-\lambda_{i}\right)+ & \left(1-\gamma_{i}\right)^{2}-\left(1-\gamma_{0}\right)^{2} \\
& >\left(\left(1-\gamma_{0}\right)^{2}-\gamma_{i+1}^{2}\right)\left(1-\lambda_{i+1}\right)+\left(1-\gamma_{i+1}\right)^{2}-\left(1-\gamma_{0}\right)^{2}
\end{aligned}
$$

By definition

$$
\lambda_{i}>\frac{2}{\gamma_{i}+\gamma_{i+1}}>\lambda_{i+1}
$$

Furthermore,

$$
\left(1-\gamma_{0}\right)^{2}-\gamma_{i}^{2}<\left(1-\gamma_{0}\right)^{2}-\left(\gamma_{i}-1\right)^{2}<0
$$

and therefore both left and right hand sides are increasing in $\lambda$. Therefore, it suffices to show that

$$
\begin{aligned}
\left(\left(1-\gamma_{0}\right)^{2}-\gamma_{i}^{2}\right)(1-\lambda)+\left(1-\gamma_{i}\right)^{2}-\left(1-\gamma_{0}\right)^{2} & \\
& \geq\left(\left(1-\gamma_{0}\right)^{2}-\gamma_{i+1}^{2}\right)(1-\lambda)+\left(1-\gamma_{i+1}\right)^{2}-\left(1-\gamma_{0}\right)^{2}
\end{aligned}
$$

for $\lambda=\frac{2}{\gamma_{i}+\gamma_{i+1}}$. That is,

$$
\left(\left(1-\gamma_{0}\right)^{2}-\gamma_{i}^{2}\right)(1-\lambda)+\left(1-\gamma_{i}\right)^{2}-\left(1-\gamma_{0}\right)^{2} \geq\left(\left(1-\gamma_{0}\right)^{2}-\gamma_{i+1}^{2}\right)(1-\lambda)+\left(1-\gamma_{i+1}\right)^{2}-\left(1-\gamma_{0}\right)^{2} .
$$


Equivalently,

$$
\left(\gamma_{i+1}^{2}-\gamma_{i}^{2}\right)(1-\lambda) \geq\left(\gamma_{i+1}-1\right)^{2}-\left(\gamma_{i}-1\right)^{2}
$$

Simplifying, we get that it suffices to show

$$
\lambda \leq \frac{2}{\gamma_{i}+\gamma_{i+1}}
$$

which is true.

Proof of Corollary 5.1: We only have to show that $R(\lambda)$ is decreasing. By the definition of $\gamma_{0}$,

$$
\left(1-\gamma_{i}\right)^{2}>\left(1-\gamma_{0}\right)^{2}
$$

and therefore $R(\lambda)$ is monotone decreasing in $\lambda$ on each $\Pi_{i}$. Thus, it remains to show that $R(\lambda)$ is continuous at the boundaries of $\Pi_{i}$. Denote by $R_{i}(\lambda)$ the restriction of $R(\lambda)$ onto $\Pi_{i}$. A direct calculation shows that

$$
R_{i+1}\left(\frac{2}{\gamma_{i}+\gamma_{i+1}}\right)=R_{i}\left(\frac{2}{\gamma_{i}+\gamma_{i+1}}\right)
$$

and we are done.

Lemma C.5 The function $U^{\prime}(x) x^{\gamma_{-k}}$ is monotone decreasing in $x$ and $U^{\prime}(x) x^{\gamma_{l}}$ is monotone increasing in $x$.

Proof: We have

$$
\left(U^{\prime}(x) x^{\alpha}\right)^{\prime}=U^{\prime \prime}(x) x^{\alpha}+\alpha U^{\prime}(x) x^{\alpha-1}=x^{\alpha-1} U^{\prime}(x)\left(\alpha-\gamma^{U}(x)\right)
$$

for any $\alpha \in \mathbb{R}$ and the claim follows from Proposition 6.3.

Proof of Proposition 5.1: We need to show that

$$
\frac{E_{t}\left[D^{1-\gamma_{l}}\right]}{E_{t}\left[D^{-\gamma_{l}}\right]} \leq \frac{E_{t}[D M]}{E_{t}[M]} \leq \frac{E_{t}\left[D^{1-\gamma_{-k}}\right]}{E_{t}\left[D^{-\gamma_{-k}}\right]}
$$

We will only prove the lower bound. The upper bound is proved similarly.

Introduce a new measure

$$
d Q=\frac{D^{-\gamma_{l}} d P}{E_{t}\left[D^{-\gamma_{k}}\right]} .
$$

Then, under this new measure, the required inequality takes the form

$$
\frac{E_{t}^{Q}\left[D\left(M D^{\gamma_{l}}\right)\right]}{E_{t}^{Q}\left[M D^{\gamma_{l}}\right]} \geq E_{t}^{Q}[D] .
$$

This inequality follows from Lemmas C.5 and 4.2. 


\section{Proofs for Section 6}

Proof of Proposition 6.3: Let

$$
z_{i}=\psi_{i} E[D M] E\left[M^{1-b_{i}}\right]^{-1}
$$

Differentiating (16), we get

$$
U^{\prime \prime}(D)=-\frac{1}{\sum_{i} z_{i} b_{i} M^{-b_{i}-1}} \Rightarrow \gamma^{U}(D)=\frac{D}{\sum_{i} z_{i} b_{i} M^{-b_{i}}}
$$

and therefore, using

$$
\sum_{i} z_{i} M^{-b_{i}}=D
$$

we get

$$
\begin{aligned}
& -\frac{d}{d D} \gamma^{U}(D)=-\frac{1}{\sum_{i} z_{i} b_{i} M^{-b_{i}}}+\frac{D \sum_{i} z_{i} b_{i}^{2} M^{-b_{i}}}{\left(\sum_{i} z_{i} b_{i} M^{-b_{i}}\right)^{3}} \\
& \quad=\left(\sum_{i} z_{i} b_{i} M^{-b_{i}}\right)^{-3}\left(\sum_{i} z_{i} b_{i}^{2} M^{-b_{i}} \sum_{i} z_{i} M^{-b_{i}}-\left(\sum_{i} z_{i} b_{i} M^{-b_{i}}\right)^{2}\right) .
\end{aligned}
$$

Applying the Cauchy-Schwarz inequality

$$
\sum_{i} x_{i} \sum_{i} y_{i} \geq\left(\sum_{i}\left(x_{i} y_{i}\right)^{1 / 2}\right)^{2}
$$

to the numbers

$$
x_{i}=z_{i} b_{i}^{2} M^{-b_{i}}, y_{i}=z_{i} M^{-b_{i}}
$$

we get the first statement. The second is straightforward to check.

Proof of Proposition 6.1: The first inequality follows directly from Lemma 6.1. If $\gamma_{i}>\gamma_{j}>1$, then $b_{i}<b_{j}<1$ and Lemma 6.1 implies that

$$
\left(1-b_{i}\right) \frac{E_{t}\left[M^{1-b_{i}} \gamma^{U}(D)\right]}{E_{t}\left[M^{1-b_{i}}\right]} \geq\left(1-b_{j}\right) \frac{E_{t}\left[M^{1-b_{j}} \gamma^{U}(D)\right]}{E_{t}\left[M^{1-b_{j}}\right]}
$$

that is $\pi_{i t}<\pi_{j t}$. Similarly, by Lemma 6.1,

$$
\frac{E_{t}\left[M^{1-b_{i}} \gamma^{U}(D)\right]}{E_{t}\left[M^{1-b_{i}}\right]}<\frac{E_{t}\left[M \gamma^{U}(D)\right]}{E_{t}[M]}
$$

and therefore

$$
\left(b_{i}-1\right) \frac{E_{t}\left[M^{1-b_{i}} \gamma^{U}(D)\right]}{E_{t}\left[M^{1-b_{i}}\right]}+\frac{E_{t}\left[M \gamma^{U}(D)\right]}{E_{t}[M]} \geq b_{i} \frac{E_{t}\left[M \gamma^{U}(D)\right]}{E_{t}[M]}
$$


if $\gamma_{i}>1$ and

$$
\left(b_{i}-1\right) \frac{E_{t}\left[M^{1-b_{i}} \gamma^{U}(D)\right]}{E_{t}\left[M^{1-b_{i}}\right]}+\frac{E_{t}\left[M \gamma^{U}(D)\right]}{E_{t}[M]} \leq b_{i} \frac{E_{t}\left[M \gamma^{U}(D)\right]}{E_{t}[M]}
$$

if $\gamma_{i}<1$. Finally, the last inequality follows from

$$
\min _{i} \gamma_{i} \leq \gamma^{U}(D) \leq \max _{i} \gamma_{i}
$$

Proof of Proposition 6.4. Recall that price $S_{t}$ and the wealth of fund $k$ satisfy

$$
\begin{gathered}
\log S_{t}=\log E_{t}[M D]-\log E_{t}[M] \\
\log W_{k t}=\log \left(\frac{\psi_{k} E[D M]}{E\left[M^{1-b_{k}}\right]} E_{t}\left[M^{1-b_{k}}\right]\right)-\log E_{t}[M] .
\end{gathered}
$$

We get the volatility $\sigma_{t}$ as the Malliavin derivative $\mathcal{D}_{t} \log S_{t}$ and we get $\sigma_{t} \pi_{k t}$ as the Malliavin derivative $\mathcal{D}_{t} \log W_{k t} \cdot{ }^{13}$ Thus, we have

$$
\pi_{k t}=\frac{\mathcal{D}_{t} \log W_{k t}}{\mathcal{D}_{t} \log S_{t}}
$$

We will now calculate the Malliavin derivatives. Since $D=e^{\rho T+\sigma B_{T}}$, we have

$$
\mathcal{D}_{t} D=\sigma D
$$

and therefore, using (15)-(16), we get

$$
\mathcal{D}_{t} M=U^{\prime \prime}(D) \mathcal{D}_{t} D=\sigma D U^{\prime \prime}(D)
$$

Using the identity

$$
\mathcal{D}_{t} E_{t}[X]=E_{t}\left[\mathcal{D}_{t} X\right]
$$

we can compute

$$
\mathcal{D}_{t} \log W_{k t}=\frac{1-b_{i}}{E_{t}\left[M^{1-b_{i}}\right]} E_{t}\left[M^{-b_{i}} \mathcal{D}_{t} M\right]-\frac{1}{E_{t}[M]} E_{t}\left[\mathcal{D}_{t} M\right]
$$

and

$$
\begin{aligned}
\mathcal{D}_{t} \log S_{t}=\frac{1}{E_{t}[M D]} E_{t}\left[D \mathcal{D}_{t} M+\sigma D M\right]-\frac{1}{E_{t}[M]} & E_{t}\left[\mathcal{D}_{t} M\right] \\
& =\sigma+\frac{E_{t}\left[D \mathcal{D}_{t} M\right]}{E_{t}[D M]}-\frac{E_{t}\left[\mathcal{D}_{t} M\right]}{E_{t}[M]} .
\end{aligned}
$$

\footnotetext{
${ }^{13}$ For an expedient introduction to Malliavin derivatives see Detemple, Garcia and Rindisbacher (2003).
} 
It remains to show the expression for the drift. By the above,

$$
\frac{d E_{t}[M D]}{E_{t}[M D]}=U_{t} d W_{t}, \frac{d E_{t}[M]}{E_{t}[M]}=V_{t} d W_{t}
$$

where, by (65),

$$
U_{t}=\frac{\mathcal{D}_{t} E_{t}[M D]}{E_{t}[M D]}=\sigma\left(1-\frac{E_{t}\left[\gamma^{U}(D) D M\right]}{E_{t}[D M]}\right)
$$

and

$$
V_{t}=\frac{\mathcal{D}_{t} E_{t}[M]}{E_{t}[M]}=-\sigma \frac{E_{t}\left[\gamma^{U}(D) M\right]}{E_{t}[M]}
$$

Applying Ito's formula, we get

$$
d \log S_{t}=r d t+d \log \frac{E_{t}[M D]}{E_{t}[M]}=\frac{1}{2}\left(2 r+V_{t}^{2}-U_{t}^{2}\right) d t+\left(U_{t}-V_{t}\right) d W_{t} .
$$

Therefore,

$$
\mu_{t}=r+\frac{1}{2}\left(V_{t}^{2}-U_{t}^{2}+\left(U_{t}-V_{t}\right)^{2}\right)=r+V_{t}\left(V_{t}-U_{t}\right)
$$

and the claim follows.

Proof of Lemma 6.1: Introduce the new measure

$$
d Q=\frac{M}{E[M]} d P
$$

Then,

$$
\frac{E_{t}\left[M \gamma^{U}(D)\right]}{E_{t}[M]}=E_{t}^{Q}\left[\gamma^{U}(D)\right], \frac{E_{t}\left[M D \gamma^{U}(D)\right]}{E_{t}[M D]}=\frac{E_{t}^{Q}\left[D \gamma^{U}(D)\right]}{E_{t}^{Q}[D]} .
$$

Lemmas 4.2 and 6.3 imply

$$
E_{t}^{Q}\left[\gamma^{U}(D)\right] E_{t}^{Q}[D] \geq E_{t}^{Q}\left[D \gamma^{U}(D)\right]
$$

and the claim follows.

Let now $x>y$ and consider the new measure

$$
d Q=\frac{M^{1-x}}{E\left[M^{1-x}\right]} d P
$$

Then,

$$
\frac{E_{t}\left[M^{1-x} \gamma^{U}(D)\right]}{E_{t}\left[M^{1-x}\right]}=E_{t}^{Q}\left[\gamma^{U}(D)\right]
$$

and

$$
\frac{E_{t}\left[M^{1-y} \gamma^{U}(D)\right]}{E_{t}\left[M^{1-y}\right]}=\frac{E_{t}^{Q}\left[M^{x-y} \gamma^{U}(D)\right]}{E_{t}^{Q}\left[M^{x-y}\right]} .
$$

Since $M$ is monotone decreasing in $D$, the variable $M^{x-y}$ is also decreasing in $D$ and the claim follows from Lemmas 4.2 and 6.3. 
Proof of Lemma 6.2:

We have

$$
\begin{aligned}
& \gamma^{U}(D)-\gamma_{J(1-\alpha)}=\frac{D}{\sum_{i} \psi_{i} E[D M] E\left[M^{1-b_{i}}\right]^{-1} b_{i} M^{-b_{i}}}-\gamma_{J(1-\alpha)} \\
& =\frac{\sum_{i} \psi_{i} E[D M] E\left[M^{1-b_{i}}\right]^{-1} M^{-b_{i}}}{\sum_{i} \psi_{i} E[D M] E\left[M^{1-b_{i}}\right]^{-1} b_{i} M^{-b_{i}}}-\gamma_{J(1-\alpha)} \frac{\sum_{i} \psi_{i} E[D M] E\left[M^{1-b_{i}}\right]^{-1} b_{i} M^{-b_{i}}}{\sum_{i} \psi_{i} E[D M] E\left[M^{1-b_{i}}\right]^{-1} b_{i} M^{-b_{i}}} \\
& =\frac{\sum_{i \neq J(1-\alpha)} \psi_{i} E[D M] E\left[M^{1-b_{i}}\right]^{-1}\left(1-b_{i} \gamma_{J(1-\alpha)}\right) M^{-b_{i}}}{\sum_{i} \psi_{i} E[D M] E\left[M^{1-b_{i}}\right]^{-1} b_{i} M^{-b_{i}}}=\frac{\sum_{i \neq J(1-\alpha)}\left(1-b_{i} \gamma_{J(1-\alpha)}\right) W_{i T}}{\sum_{i} b_{i} W_{i T}} .
\end{aligned}
$$

We have

$$
\min _{i} b_{i} D \leq \sum_{i} b_{i} W_{i T} \leq \max _{i} b_{i} D
$$

and therefore

$$
\left|\gamma^{U}(D)-\gamma_{J(1-\alpha)}\right| \leq K \sum_{i \neq J(1-\alpha)} W_{i T} D^{-1} .
$$

Thus, it suffices to show that

$$
\frac{E_{t}\left[W_{i T} D^{\alpha-1}\right]}{E_{t}\left[D^{\alpha}\right]} \rightarrow 0
$$

for all $i \neq J(1-\alpha)$.

Applying Lemma 4.4, we get

$$
E[D M]^{1-\gamma_{i}} E\left[M^{1-b_{i}}\right]^{\gamma_{i}} \geq K E\left[D^{1-\gamma_{i}}\right]
$$

that is

$$
E[D M] E\left[M^{1-b_{i}}\right]^{-1} \leq E[D M]^{b_{i}} K^{-b_{i}} E\left[D^{1-\gamma_{i}}\right]^{-b_{i}}
$$

Similarly,

$$
\left(\left(\psi_{i} E[D M] / E\left[M^{1-b_{J(1-\alpha)}}\right]\right)^{\gamma_{J(1-\alpha)}} \leq K E[D M] E\left[D^{1-\gamma_{J(1-\alpha)}}\right]^{-1}\right.
$$

By Lemma 2.1,

$$
M \geq\left(\psi_{i} E[D M] / E\left[M^{1-b_{J(1-\alpha)}}\right]\right)^{\gamma_{J(1-\alpha)}} D^{-\gamma_{J(1-\alpha)}}
$$

and hence,

$$
\begin{aligned}
W_{i T}= & \psi_{i} E[D M] E\left[M^{1-b_{i}}\right]^{-1} M^{-b_{i}} \leq \\
& \psi_{i} E[D M] E\left[M^{1-b_{i}}\right]^{-1}\left(\left(\psi_{i} E[D M] / E\left[M^{1-b_{J(1-\alpha)}}\right]\right)^{\gamma_{J(1-\alpha)}} D^{-\gamma_{J(1-\alpha)}}\right)^{-b_{i}} \\
& \leq K \frac{E\left[D^{1-\gamma_{J(1-\alpha)}}\right]^{b_{i}}}{E\left[D^{1-\gamma_{i}}\right]^{b_{i}}} D^{\gamma_{J(1-\alpha)} b_{i}}
\end{aligned}
$$

Therefore,

$$
W_{i T} D^{-1} \leq K e^{\frac{1}{2} \sigma^{2} T b_{i}\left(\left(1-\gamma_{J(1-\alpha)}\right)^{2}-\left(1-\gamma_{i}\right)^{2}\right)} e^{\sigma\left(\gamma_{J(1-\alpha)} b_{i}-1\right) B_{T}} .
$$


Introduce now a new family of measures

$$
d Q_{t}=\frac{D_{t}^{\alpha} d P_{t}}{E\left[D_{t}^{\alpha}\right]}
$$

of $\mathcal{F}_{t}$, It is not difficult to see that this family of measures is time consistent and hence, by the Kolmogorov theorem (Oksendal (2003), p.11), there is a unique measure $d Q$ on inifinitehorizon paths generating them on finite horizon $\sigma$-algebras $\mathcal{F}_{t}$. Under this measure, $\sigma B_{T}$ will also be a Brownian motion, but with a drift. Namely,

$$
E^{Q}\left[e^{\sigma B_{T}}\right]=\frac{E\left[e^{(\alpha+1) \sigma B_{T}}\right]}{E\left[e^{\alpha \sigma B_{T}}\right]}=e^{\frac{1}{2} \sigma^{2} T(2 \alpha+1)}=e^{\alpha \sigma^{2} T+\frac{1}{2} \sigma^{2} T}
$$

and hence it has a drift $\alpha \sigma^{2}$. Thus, under this measure $\sigma B_{T}=\alpha \sigma^{2} T+\sigma B_{T}^{Q}$ and

$$
\begin{aligned}
& e^{\frac{1}{2} \sigma^{2} T b_{i}\left(\left(1-\gamma_{J(1-\alpha)}\right)^{2}-\left(1-\gamma_{i}\right)^{2}\right)} e^{\sigma\left(\gamma_{J(1-\alpha)} b_{i}-1\right) B_{T}} \\
& \quad=e^{\frac{1}{2} \sigma^{2} T\left(b_{i}\left(\left(1-\gamma_{J(1-\alpha)}\right)^{2}-\left(1-\gamma_{i}\right)^{2}\right)+2 \alpha\left(\gamma_{J(1-\alpha)} b_{i}-1\right)\right)} e^{\sigma\left(\gamma_{J(1-\alpha)} b_{i}-1\right) \sigma B_{T}^{Q}} .
\end{aligned}
$$

The most important observation is that

$$
\begin{aligned}
b_{i}\left(\left(1-\gamma_{J(1-\alpha)}\right)^{2}-\left(1-\gamma_{i}\right)^{2}\right) & +2 \alpha\left(\gamma_{J(1-\alpha)} b_{i}-1\right)<0 \\
\Leftrightarrow & \Leftrightarrow\left(1-\gamma_{J(1-\alpha)}\right)^{2}-\left(1-\gamma_{i}\right)^{2}+2 \alpha\left(\gamma_{J(1-\alpha)}-\gamma_{i}\right)<0 .
\end{aligned}
$$

In fact,

$$
\begin{aligned}
\left(1-\gamma_{J(1-\alpha)}\right)^{2}+2 \alpha \gamma_{J(1-\alpha)} & =\left(\gamma_{J(1-\alpha)}-(1-\alpha)\right)^{2}-(1-\alpha)^{2}+1 \\
< & \left(\gamma_{i}-(1-\alpha)\right)^{2}-(1-\alpha)^{2}+1=\left(1-\gamma_{i}\right)^{2}+2 \alpha \gamma_{i}
\end{aligned}
$$

which follows from the definition of $J(1-\alpha)$.

Let now

$$
\epsilon=\frac{1}{2} \sigma^{2}\left(b_{i}\left(-\left(1-\gamma_{J(1-\alpha)}\right)^{2}+\left(1-\gamma_{i}\right)^{2}\right)-2 \alpha\left(\gamma_{J(1-\alpha)} b_{i}-1\right)\right)>0 .
$$

Let also $\gamma_{J(1-\alpha)} b_{i}>1$ (the case $\gamma_{J(1-\alpha)} b_{i}<1$ is completely analogous). Then, since $W_{i T} D^{-1} \leq 1$

$$
\begin{aligned}
& E_{t}^{Q}\left[W_{i T} D^{-1}\right]=E_{t}^{Q}\left[W_{i t} D^{-1} I_{\sigma B_{T}^{Q}<\epsilon T /\left(2\left(\gamma_{J(1-\alpha)} b_{i}-1\right)\right)}\right] \\
& +E_{t}^{Q}\left[W_{i T} D^{-1} I_{\sigma B_{T}^{Q}>\epsilon T /\left(2\left(\gamma_{J(1-\alpha)} b_{i}-1\right)\right)}\right] \leq K e^{-\epsilon T / 2}+E_{t}^{Q}\left[I_{\sigma B_{T}^{Q}>\epsilon T /\left(2\left(\gamma_{J(1-\alpha)} b_{i}-1\right)\right)}\right] .
\end{aligned}
$$

Let

$$
\delta=\sigma^{-1} \epsilon /\left(2\left(\gamma_{J(1-\alpha)} b_{i}-1\right)\right)>0
$$


Then,

$$
\begin{aligned}
E_{t}^{Q}\left[I_{\sigma B_{T}^{Q}>\epsilon T /\left(2\left(\gamma_{J(1-\alpha)} b_{i}-1\right)\right)}\right]=\operatorname{Prob}_{t}^{Q}\left[\sigma\left(B_{T}^{Q}-B_{t}^{Q}\right) \geq T \sigma\left(\delta-B_{t}^{Q} / T\right)\right] \\
=\left(1-N\left(\sigma \sqrt{T}\left(\delta-B_{t}^{Q} /\left(t \lambda^{-1}\right)\right) /(\sigma \sqrt{1-\lambda})\right)\right) .
\end{aligned}
$$

By the Law of Large Numbers, $B_{t}^{Q} / t \rightarrow 0$ as $t \rightarrow \infty$ and therefore

$$
\delta-\frac{B_{t}^{Q}}{t \lambda^{-1}} \rightarrow \delta>0
$$

almost surely. Hence,

$$
\sqrt{T}\left(\delta-B_{t}^{Q} /\left(t \lambda^{-1}\right)\right) /(\sigma \sqrt{1-\lambda}) \rightarrow+\infty
$$

as $T \rightarrow \infty$ and

$$
N\left(\sigma \sqrt{T}\left(\delta-B_{t}^{Q} /\left(t \lambda^{-1}\right)\right) /(\sigma \sqrt{1-\lambda})\right) \rightarrow 1
$$

Therefore,

$$
E_{t}\left[I_{\sigma B_{T}^{Q}>\epsilon T /\left(2\left(\gamma_{J(1-\alpha)} b_{i}-1\right)\right)}\right] \rightarrow 0
$$

and the claim follows.

The proof for a fixed, finite $t$ is analogous. In fact, in this case the claim also follows from almost sure convergence and the Lebesque dominated convergence theorem.

Proof of Proposition 6.5: It is a direct consequence of Lemma 6.2 and Lemma D.1 below.

Lemma D.1 We have

$$
\frac{E_{t}\left[M D \gamma^{U}(D)\right]}{E_{t}[M D]} \sim \frac{E_{t}\left[D^{1-\gamma_{0}} \gamma^{U}(D)\right]}{E_{t}\left[D^{1-\gamma_{0}}\right]}
$$

and, for $\lambda \in \Pi_{i}$,

$$
\frac{E_{t}\left[M \gamma^{U}(D)\right]}{E_{t}[M]} \sim \frac{E_{t}\left[D^{-\gamma_{i}} \gamma^{U}(D)\right]}{E_{t}\left[D^{-\gamma_{i}}\right]}
$$

Proof: In complete analogy with (52), we have

$$
\begin{aligned}
\left(\sum_{i=1}^{n} E_{t}\left[\gamma^{U}(D) D^{-\gamma_{i}}\right]^{1 / \gamma}(\right. & \left.\left.\psi_{i} E[D M] / E\left[M^{1-b_{i}}\right]\right)^{\gamma_{i} / \gamma}\right)^{\gamma} \leq E_{t}\left[M \gamma^{U}(D)\right] \\
& \leq\left(\sum_{i} E_{t}\left[\gamma^{U}(D) D^{-\gamma_{i}}\right]^{1 / \Gamma}\left(\psi_{i} E[D M] / E\left[M^{1-b_{i}}\right]\right)^{\gamma_{i} / \Gamma}\right)^{\Gamma} .
\end{aligned}
$$

Since $\gamma^{U}(D)$ is bounded away from zero and infinity, the same reasoning as in the proof of Lemma C.4 implies

$$
\lim _{T \rightarrow \infty} \frac{E_{t}\left[M \gamma^{U}(D)\right]}{\left(\psi_{i} E[D M] E\left[M^{1-b_{i}}\right]^{-1}\right)^{\gamma_{i}} E_{t}\left[\gamma^{U}(D) D^{-\gamma_{i}}\right]}=1
$$


for $\lambda \in \Pi_{i}$. Similarly, the same reasoning as in the proof of Lemma C.3 implies that

$$
\lim _{T \rightarrow \infty} \frac{E_{t}\left[M D \gamma^{U}(D)\right]}{\left(\psi_{0} E[D M] E\left[M^{1-b_{0}}\right]^{-1}\right)^{\gamma_{0}} E_{t}\left[\gamma^{U}(D) D^{1-\gamma_{0}}\right]}=1 .
$$

Lemma D.2 Assume $\gamma_{i}>1$. Let $\Gamma \geq 1$ be such that $\Gamma b_{k} /\left(1-b_{i}\right)>1$ for all $k$ and $\gamma \leq 1$ be such that $\gamma b_{k} /\left(1-b_{i}\right) \leq 1$ for all $k$. Then,

$$
\begin{gathered}
\left(\sum_{k} D^{-\gamma_{k}\left(1-b_{i}\right) / \gamma}\left(\psi_{k} E[D M] / E\left[M^{1-b_{k}}\right]\right)^{\gamma_{k}\left(1-b_{i}\right) / \gamma}\right)^{\gamma} \leq M^{1-b_{i}} \\
\leq\left(\sum_{k} D^{-\gamma_{k}\left(1-b_{i}\right) / \Gamma}\left(\psi_{k} E[D M] / E\left[M^{1-b_{k}}\right]\right)^{\gamma_{k}\left(1-b_{i}\right) / \Gamma}\right)^{\Gamma} .
\end{gathered}
$$

The proof is completely analogous to that of Lemma 4.3 after rewriting the equilibrium equation as

$$
\sum_{k} z_{k}\left(M^{1-b_{i}}\right)^{-b_{k} /\left(1-b_{i}\right)}=D
$$

with

$$
z_{k}=\psi_{k} E[D M] / E\left[M^{1-b_{k}}\right]
$$

Lemma D.3 For $\lambda \in \Theta_{j}^{i}$,

$$
E_{t}\left[M^{1-b_{i}}\right] \sim\left(\psi_{j} E[D M] E\left[M^{1-b_{j}}\right]^{-1}\right)^{\gamma_{j}\left(1-b_{i}\right)} E_{t}\left[D^{-\gamma_{j}\left(1-b_{i}\right)}\right]
$$

and

$$
E_{t}\left[M^{1-b_{i}} \gamma^{U}(D)\right] \sim\left(\psi_{j} E[D M] E\left[M^{1-b_{j}}\right]^{-1}\right)^{\gamma_{j}\left(1-b_{i}\right)} E_{t}\left[\gamma^{U}(D) D^{-\gamma_{j}\left(1-b_{i}\right)}\right]
$$

Proof: In complete analogy with (59), we get

$$
\begin{gathered}
\left(1+K_{1} \sum_{k \neq j}\left(\frac{E_{t}\left[D^{-\gamma_{k}\left(1-b_{i}\right)}\right] E\left[D^{1-\gamma_{j}}\right]^{1-b_{i}}}{E_{t}\left[D^{-\gamma_{j}\left(1-b_{i}\right)}\right] E\left[D^{1-\gamma_{k}}\right]^{1-b_{i}}}\right)^{1 / \gamma}\right)^{\gamma} \\
\leq \frac{E_{t}\left[M^{1-b_{i}}\right]}{\left(\psi_{j} E[D M] E\left[M^{1-b_{j}}\right]^{-1}\right)^{\gamma_{j}\left(1-b_{i}\right)} E_{t}\left[D^{-\gamma_{j}\left(1-b_{i}\right)}\right]} \\
\leq\left(1+K_{1} \sum_{k \neq j}\left(\frac{E_{t}\left[D^{-\gamma_{k}\left(1-b_{i}\right)}\right] E\left[D^{1-\gamma_{j}}\right]^{1-b_{i}}}{E_{t}\left[D^{-\gamma_{j}\left(1-b_{i}\right)}\right] E\left[D^{1-\gamma_{k}}\right]^{1-b_{i}}}\right)^{1 / \Gamma}\right)^{\Gamma} .
\end{gathered}
$$

Now,

$$
\left.\frac{E_{t}\left[D^{-\gamma_{k}\left(1-b_{i}\right)}\right] E\left[D^{1-\gamma_{j}}\right]^{1-b_{i}}}{E_{t}\left[D^{-\gamma_{j}\left(1-b_{i}\right)}\right] E\left[D^{1-\gamma_{k}}\right]^{1-b_{i}}}=e^{\frac{1}{2} \sigma^{2} T\left(1-b_{i}\right)\left((1-\lambda)\left(\gamma_{k}^{2}-\gamma_{j}^{2}\right)\left(1-b_{i}\right)+\left(1-\gamma_{j}\right)^{2}-\left(1-\gamma_{k}\right)^{2}\right.}\right)
$$


and we need to show that

$$
(1-\lambda) \gamma_{k}^{2}\left(1-b_{i}\right)-\left(1-\gamma_{k}\right)^{2}<(1-\lambda) \gamma_{j}^{2}\left(1-b_{i}\right)-\left(1-\gamma_{j}\right)^{2}
$$

The function

$$
f(\gamma)=(1-\lambda) \gamma^{2}\left(1-b_{i}\right)-(1-\gamma)^{2}=-\left(1-(1-\lambda)\left(1-b_{i}\right)\right) \gamma^{2}+2 \gamma-1
$$

is a quadratic parabola with the vertex at $\gamma^{v}=1 /\left(1-(1-\lambda)\left(1-b_{i}\right)\right)$. Therefore, the inequality holds if and only if

$$
\left|\gamma_{k}-\gamma^{v}\right|>\left|\gamma_{j}-\gamma^{v}\right|
$$

and the claim follows from the definition of the interval $\Theta_{j}^{i}$.

Proof of Lemma 6.4. Introducing the new measure

$$
d Q=\frac{D^{-\gamma-k}}{E_{t}\left[D^{-\gamma-k}\right]} d P
$$

we can rewrite the required inequality

$$
\frac{E_{t}\left[D^{-\gamma_{-k} \alpha} \gamma^{U}(D)\right]}{E_{t}\left[D^{-\gamma_{-k} \alpha}\right]} \leq \frac{E_{t}\left[M^{\alpha} \gamma^{U}(D)\right]}{E_{t}\left[M^{\alpha}\right]}
$$

in the form

$$
E_{t}^{Q}\left[\gamma^{U}(D)\right] \leq \frac{E_{t}^{Q}\left[\left(M D^{\gamma-k}\right)^{\alpha} \gamma^{U}(D)\right]}{E_{t}\left[\left(M D^{\gamma-k}\right)^{\alpha}\right]}
$$

This inequality follows from Lemmas C.5 and 4.2.

Proof of Proposition 6.9. We have

$$
\begin{aligned}
& \pi_{\gamma}(\lambda)=\frac{\left(\gamma^{-1}-1\right)\left(1+\gamma_{j}\left(1-\gamma^{-1}\right)\right)+1+\gamma_{m}}{2+\gamma_{m}} \\
&=\frac{\left(\gamma^{-1}-1\right)\left(1+\frac{\gamma-1}{\lambda(\gamma-1)+1}\left(1-\gamma^{-1}\right)\right)+1+\lambda^{-1}}{1+\lambda^{-1}} \\
&=\frac{\lambda^{2} \gamma(\gamma-1)+\left(2\left(\gamma^{2}-\gamma\right)+1\right) \lambda+\gamma^{2}}{\gamma^{2}(\gamma-1) \lambda^{2}+\gamma^{3} \lambda+\gamma^{2}}
\end{aligned}
$$

It remains to prove monotonicity. Differentiating w.r.t. $\lambda$, we need to check that

$$
0>(\gamma-1)\left(\gamma^{2}(\gamma-1) \lambda^{2}+\gamma^{3} \lambda+\gamma^{2}\right)-((\gamma-1) \lambda+\gamma)\left(2 \gamma^{2}(\gamma-1) \lambda+\gamma^{3}\right)=-\gamma^{2}\left((\gamma-1)^{2} \lambda+\gamma\right)^{2}
$$

Proof of Proposition 6.10. Clearly, $J\left(1+\gamma_{1}\right)=1$. By Theorem 6.1, for $\lambda \in \Pi_{1}$ we have

$$
\lim _{T \rightarrow \infty} \sigma_{t}=\sigma\left(1+\gamma_{1}-\gamma_{0}\right), \lim _{T \rightarrow \infty} \mu_{t}=r+\gamma_{1}\left(1+\gamma_{1}-\gamma_{0}\right) \sigma^{2}
$$


But, for $\lambda \in \Pi_{0}$, two cases are possible. If $\gamma_{1}<2+\gamma_{0}$ then $J\left(1+\gamma_{0}\right)=\gamma_{1}$ and

$$
\lim _{T \rightarrow \infty} \sigma_{t}=\sigma\left(1+\gamma_{1}-\gamma_{0}\right), \lim _{T \rightarrow \infty} \mu_{t}=r+\gamma_{1}\left(1+\gamma_{1}-\gamma_{0}\right) \sigma^{2}
$$

If $\gamma_{1}>2+\gamma_{0}$, then $J\left(1+\gamma_{0}\right)=0$ and

$$
\lim _{T \rightarrow \infty} \sigma_{t}=\sigma, \lim _{T \rightarrow \infty} \mu_{t}=r+\gamma_{0} \sigma^{2},
$$

that is the drift and volatility agree with the asymptotic behavior, predicted by Theorem 5.1 .

By definition, $J\left(1+\gamma_{1}\left(1-b_{1}\right)\right)=J\left(\gamma_{1}\right)=1$. Furthermore,

$$
\mid\left(1+\gamma_{0}\left(1-b_{1}\right)-\gamma_{0}\left|=\frac{\gamma_{1}-\gamma_{0}}{\gamma_{1}}<\frac{\left(\gamma_{1}-\gamma_{0}\right)}{\gamma_{1}}\left(\gamma_{1}-1\right)=\right|\left(1+\gamma_{0}\left(1-b_{1}\right)-\gamma_{1} \mid,\right.\right.
$$

if and only if $\gamma_{1}>2$. That is $J\left(1+\gamma_{0}\left(1-b_{1}\right)\right)=1$ for $\gamma_{1}<2$ and is 0 otherwise. Then, Theorem 6.2 implies the required expressions.

\section{References}

[1] Anderson, R. and Raimondo, R. (2008). Equilibrium in Continuous-Time Financial Markets: Endogenously Dynamically Complete Markets. Econometrica,76(4), 841907.

[2] Benninga, S. and Mayshar, J. (2000). Heterogeneity and Option Pricing. Review of Derivatives Research, 4(1): 7-27.

[3] Berrada, T. (2008). Bounded Rationality and Asset Pricing with Intermediate Consumption. Review of Finance, forthcoming.

[4] Blume, L., and Easley, D. (1992). Evolution and Market Behavior. Journal of Economic Theory, 58(1), 9-40.

[5] Blume, L., and Easley, D. (2006). If you are so smart, why aren't you rich. Belief selection in complete and incomplete markets. Econometrica, 74(4), 929-966.

[6] Cao, H. H. and Ou-Yang, H. (2005). Bubbles and Panics in a Frictionless Market with Heterogeneous Expectations. Working paper.

[7] Cvitanić, J. and Zapatero, F. (2004) Introduction to the Economics and Mathematics of Financial Markets. The MIT Press, Cambridge, Massachusetts. 
[8] Dana, R. (1995). An extension of Milleron, Mitjushin and Polterovich's result, Journal of Mathematical Economics, 22, 1995.

[9] Dana, R. (2001). Risk aversion and uniqueness of equilibrium. An application to financial markets" Review of Economic Design 6, 155-173, special issue in the honor of R. Radner, edited by Ischiishi.T. et Marshak T.

[10] Detemple, J.B., Garcia, R. and Rindisbacher, M. (2003) A Monte Carlo Method for Optimal Portfolios. The Journal of Finance, 58, $401-446$.

[11] Duffie, D. and Huang, C. (1986). Implementing Arrow-Debreu Equilibria by Continuous Trading of Few Long-Lived Securities. Econometrica, 53 (6), 133756.

[12] Duffie, D. (2001). Dynamic Asset Pricing Theory, Third Edition, Princeton University Press.

[13] Dybvig, P. H., Rogers, L. C. G., and K. Back. (1999). Portfolio Turnpikes. The Review of Financial Studies 12, 165-195.

[14] Guasoni, P. and Robertson, S. (2008). Portfolios and Risk Premia for the Long Run. Working paper, Boston University.

[15] Hansen, L. P., Jagannathan R. (1991). Implications of security market data for models of dynamic economies. Journal of Political Economy 99, 225-262.

[16] Huang, C., and Zariphopoulou, T. (1999). Turnpike behavior of long-term investments. Finance and Stochastics 3, 15-34.

[17] Evstigneev, I. V., Hens, T. and Schenk-Hoppe, K. R. (2006). Evolutionary Stable Markets. Economic Theory 27(2), 2006, 449-468.

[18] Jouini, E. and Napp, C. (2008). Consensus Consumer and Intertemporal Asset Pricing with Heterogeneous Beliefs. Forthcoming in the Review of Financial Studies.

[19] I. Karatzas, S. Shreve, Methods of Mathematical Finance, Springer-Verlag, New York, 1998.

[20] Kogan, L., Ross, S., Wang, J. and Westerfield, M. (2006). The Price Impact and Survival of Irrational Traders. Journal of Finance, 61(1), 195-229.

[21] Malamud, S. (2008). Long run forward rates and long yields of bonds and options in heterogeneous equilibria, Finance and Stochastics, 12, 245-264 
[22] Malamud, S. (2008). Universal bounds for asset prices in heterogeneous economies, Finance and Stochastics (2008), 12, 411-422.

[23] Merton, R. (1971). Optimum Consumption and Portfolio Rules in a Continuous Time Model. Journal of Economic Theory 3, 373-413.

[24] Shefrin, H. (2005). A Behavioral Approach to Asset Pricing. Boston: Elsevier Academic Press.

[25] Oksendal, Bernt (2003). Stochastic Differential Equations: An Introduction with Applications. Springer, Berlin.

[26] Yan, Hongjun (2009). Natural Selection in Financial Markets: Does It Work? Management Science, forthcoming.

[27] Wang, J. (1996). The Term Structure of Interest Rates In A Pure Exchange Economy With Heterogeneous Investors. Journal of Financial Economics 41, 75-110. 